\title{
Supplemental Material \\ Operationally Unsaturated Pincer/Rhenium Complexes Form Metal Carbenes From Cycloalkenes and Metal Carbynes From Alkanes
}

Oleg V. Ozerov ${ }^{1}$, Lori A. Watson ${ }^{2}$, Maren Pink, and Kenneth G. Caulton*

\section{Computational Details:}

All calculations were performed with the Gaussian 98 package. ${ }^{\text {i }}$ All calculations were preformed at the B3PW91 ${ }^{\mathrm{ii}}$ level of theory, with unrestricted wavefunctions used for all triplet state calculations. Basis sets used included LANL2DZ for Re and Si, 6-31G ${ }^{*}$ for $\mathrm{C}$ and $\mathrm{N}$, and $6-31 \mathrm{G}^{* *}$ for all hydrogens. ${ }^{\text {iii }}$ The basis set LANL2DZ is the Los Alamos National Laboratory ECP plus a double zeta valence on $\mathrm{Re}, \mathrm{P}$ and $\mathrm{Si} ;{ }^{\text {iv }}$ additional $\mathrm{d}$ polarization functions ${ }^{\mathrm{v}}$ were added to all phosphorus and silicon atoms in all DFT calculations. All optimizations were performed with $\mathrm{C}_{1}$ symmetry and all minima were confirmed by analytical calculation of frequencies, which were also used to compute zero point energy corrections without scaling.

Benzene C-H Cleavage. (PNP) $\mathrm{Re}(\mathrm{H})_{4}$ neither reacts with, nor exchanges $\mathrm{H}$ with $\mathrm{D}$ when dissolved in $\mathrm{C}_{6} \mathrm{D}_{6}$ at $22{ }^{\circ} \mathrm{C}$. This is in agreement with the $\Delta \mathrm{G}^{\mathrm{o}}{ }_{298}$ in eq. 4. Similarly, (PNP)Re(H) 2 (cyclooctyne)

$$
\begin{aligned}
& \left(\mathrm{PNP}^{\mathrm{H}}\right) \operatorname{Re}(\mathrm{H})_{4}+\mathrm{C}_{6} \mathrm{H}_{6} \stackrel{+10.7}{\longrightarrow}\left(\mathrm{PNP}^{\mathrm{H}}\right) \operatorname{Re}(\mathrm{H})_{3} \mathrm{Ph}+\mathrm{H}_{2} \\
& \left(\mathrm{PNP}^{\mathrm{H}}\right) \operatorname{Re}(\mathrm{H})_{2}(\mathrm{HCCH})+\mathrm{C}_{6} \mathrm{H}_{6} \stackrel{+39.9}{\longrightarrow}\left(\mathrm{PNP}^{\mathrm{H}}\right) \operatorname{Re}(\mathrm{H})_{3} \mathrm{Ph}+\mathrm{HC} \equiv \mathrm{CH} \\
& \left(\mathrm{PNP}^{\mathrm{H}}\right) \operatorname{Re}(\mathrm{H})_{2}\left(=\mathrm{C}\left(\mathrm{CH}_{2}\right)_{5}\right)+\mathrm{C}_{6} \mathrm{H}_{6} \stackrel{+8.5}{\longrightarrow}\left(\mathrm{PNP}^{\mathrm{H}}\right) \mathrm{Re}(\mathrm{H})_{3} \mathrm{Ph}+\text { cyclohexene }
\end{aligned}
$$

is not observed to react with benzene (eq. 5), or even to do $\mathrm{H} / \mathrm{D}$ exchange with $\mathrm{C}_{6} \mathrm{D}_{6}$ at $22{ }^{\circ} \mathrm{C}$.

However, the cyclohexylidene complex 2a does react with benzene solvent within 10 minutes at $22{ }^{\circ} \mathrm{C}$ to an equilibrium mixture of $\mathbf{2 a}$ and the benzene $\mathrm{C}-\mathrm{H}$ cleavage product $(\mathrm{PNP}) \mathrm{ReH}_{3}(\mathrm{Ph})$ and cyclohexene. The DFT-calculated thermodynamics of this reaction for the $\mathrm{PNP}^{\mathrm{H}}$ model (eq.

\footnotetext{
${ }^{1}$ Department of Chemistry, Brandeis University, 415 South Street, Waltham, MA 02454

${ }^{2}$ Department of Chemistry, Earlham College, 801 National Road West, Richmond, IN 47374
} 
6) are in satisfactory agreement with experiment (since the calculations neglect steric effects and the experiments are done far from standard state concentrations, and favor products), and make this the most thermodynamically favored of the three benzene $\mathrm{CH}$-cleavage reactions surveyed above.

Safety note. CAUTION! A number of the experiments performed here involved heating hydrocarbon solutions to elevated temperatures in a closed volume. We have repeatedly heated hydrocarbons with a boiling point of $>65{ }^{\circ} \mathrm{C}$ to $110-130{ }^{\circ} \mathrm{C}$ in closed J. Young NMR tubes (purchased from Wilmad) without accident (lower third of the tube submerged in a silicon oil bath). Nevertheless, extreme caution must be exercised. These experiments are best performed in a fume hood behind a splash shield, away from flammable and breakable materials with proper protective gear worn by the experimenter. Degassing prior to heating diminishes the pressure build-up in the system. However, this enables better heat transfer within the J. Young tube that leads to accelerated deterioration (warping) of the PTFE valve.

$\left(\mathbf{P N P}{ }^{\mathrm{iPr}}\right) \mathbf{R e H}_{2}\left(\eta^{4}\right.$-cyclohexadiene) (3). ( $\left.\mathrm{PNP}^{\mathrm{iPr}}\right) \mathrm{ReH}_{4}$ (1a) (52 mg, $\left.89 \mu \mathrm{mol}\right)$ was dissolved in $0.5 \mathrm{~mL}$ cyclohexane and $0.15 \mathrm{~mL}(1.48 \mathrm{mmol})$ cyclohexene was added to it. The mixture was heated for $2 \mathrm{~h}$ at $90{ }^{\circ} \mathrm{C}$. The volatiles were removed in vacuum, and the residue was extracted with pentane and filtered through a plug of Celite. The filtrate was palced into a $-30{ }^{\circ} \mathrm{C}$ freezer overnight. Colorless crystals of $\mathbf{3}$ were collected by decantation the next day and dried in vacuo to give $40 \mathrm{mg}(68 \%)$.

${ }^{1} \mathrm{H}$ NMR $\left(\mathrm{C}_{7} \mathrm{D}_{8}\right): \delta 4.07$ (br s, 2H, pair of $\mathrm{C}\left(\mathrm{sp}^{2}\right)-\mathrm{H}$ of $\left.\mathrm{C}_{6} \mathrm{H}_{8}\right), 2.60$ (br s, $2 \mathrm{H}$, pair of $\mathrm{C}\left(\mathrm{sp}^{2}\right)-H$ of $\left.\mathrm{C}_{6} \mathrm{H}_{8}\right), 2.26$ (br d, $2 \mathrm{H}, 9 \mathrm{~Hz}$, exo-C(sp $\left.{ }^{3}\right)-\mathrm{H}$ of $\mathrm{C}_{6} \mathrm{H}_{8}$ ), 2.00 (br m, 2H, $\mathrm{PCH}$ ), 1.75 (br m, 2H, $\mathrm{PCH}), 1.64$ (br d, 2H, $9 \mathrm{~Hz}$, endo- $\mathrm{C}\left(\mathrm{sp}^{3}\right)-\mathrm{H}$ of $\left.\mathrm{C}_{6} \mathrm{H}_{8}\right), 1.34\left(\mathrm{AB} \mathrm{dvt}, \mathrm{J}_{\mathrm{HH}}=14 \mathrm{~Hz}, \mathrm{~J}_{\mathrm{HP}}=4 \mathrm{~Hz}, 2 \mathrm{H}\right.$, $\mathrm{PCH}_{2} \mathrm{Si}$ ), 1.27 (apparent q (dvt), $\left.8 \mathrm{~Hz}, 6 \mathrm{H}, \mathrm{PCH}_{-} \mathrm{CH}_{3}\right), 0.97-1.06$ (three apparent q (dvt) 
overlapping, 18H, PCH-CH $)_{3}, 0.90\left(\mathrm{AB} d v t, \mathrm{~J}_{\mathrm{HH}}=14 \mathrm{~Hz}, \mathrm{~J}_{\mathrm{HP}}=4 \mathrm{~Hz}, 2 \mathrm{H}, \mathrm{PCH}_{2} \mathrm{Si}\right), 0.28(\mathrm{~s}, 6 \mathrm{H}$, $\left.\mathrm{SiMe}_{3}\right), 0.25$ (s, 6H, SiMe $),-8.33\left(\mathrm{br} \mathrm{dt}, J_{\mathrm{HH}}=7 \mathrm{~Hz}, J_{\mathrm{HP}}=16 \mathrm{~Hz}, \mathrm{Re}-H\right) .{ }^{1} \mathrm{H}$ NMR $\left(\mathrm{C}_{7} \mathrm{D}_{8},-75\right.$ ${ }^{\circ} \mathrm{C}$, selected resonances of the $\mathrm{C}_{6} \mathrm{H}_{8}$ ligand and $\left.\mathrm{SiMe}\right): \delta 4.62\left(\mathrm{br} \mathrm{s}, 1 \mathrm{H}, \mathrm{C}\left(\mathrm{sp}^{2}\right)-\mathrm{H}\right), 3.51$ (br s, $1 \mathrm{H}$, $\left.\mathrm{C}\left(\mathrm{sp}^{2}\right)-H\right), 2.97\left(\mathrm{~s}, 1 \mathrm{H}, \mathrm{C}\left(\mathrm{sp}^{3}\right)-H\right), 2.79\left(\mathrm{br} \mathrm{s}, 1 \mathrm{H}, \mathrm{C}\left(\mathrm{sp}^{2}\right)-H\right), 2.44\left(\mathrm{~s}, 1 \mathrm{H}, \mathrm{C}\left(\mathrm{sp}^{3}\right)-H\right), 2.08$ (br s, $\left.1 \mathrm{H}, \mathrm{C}\left(\mathrm{sp}^{2}\right)-H\right)$; SiMe peaks (all singlets): 0.48, 0.43, 0.34, 0.30 ${ }^{31} \mathrm{P}\left\{{ }^{1} \mathrm{H}\right\} \mathrm{NMR}\left(\mathrm{C}_{7} \mathrm{D}_{8}\right): \delta 29.5$ (br s). ${ }^{31} \mathrm{P}\left\{{ }^{1} \mathrm{H}\right\}$ NMR $\left(\mathrm{C}_{7} \mathrm{D}_{8},-75{ }^{\circ} \mathrm{C}\right): \delta 35.2$ (br), 26.0 (br). ${ }^{13} \mathrm{C}\left\{{ }^{1} \mathrm{H}\right\} \operatorname{NMR}\left(\mathrm{C}_{6} \mathrm{D}_{6}\right): \delta 66.2$ (br s, $C\left(\mathrm{sp}^{3}\right) \mathrm{H}_{2}$ of $\left.\mathrm{C}_{5} \mathrm{H}_{6}\right), 51.0\left(\mathrm{v}\right.$ br, $C\left(\mathrm{sp}^{2}\right) \mathrm{H}_{2}$ of $\left.\mathrm{C}_{5} \mathrm{H}_{6}\right), 31.2$ (m, PCH), 26.7 (br, $C\left(\mathrm{sp}^{2}\right) \mathrm{H}_{\text {of }} \mathrm{C}_{5} \mathrm{H}_{6}$ ), 25.5 (m, PCH), 20.7 (br s, PCHMe $), 20.6$ (br s, $\mathrm{PCHMe}_{2}$ ), 19.3 (s, $\mathrm{PCHMe}_{2}$ ), 19.1 (br s, $\mathrm{PCH}_{2} \mathrm{Si}$ ), 19.0 (s, $\mathrm{PCHMe}$ ), 7.1 (s, $\mathrm{SiMe}_{3}$ ), 6.4 (s, $\mathrm{SiMe}_{3}$ ). Compound 3. Calculated (Found) for $\mathrm{C}_{24} \mathrm{H}_{54} \mathrm{NP}_{2} \mathrm{ReSi}_{2}: \mathrm{C}, 43.61(43.71) ; \mathrm{H}, 8.23(8.25) ; \mathrm{N}, 2.12(2.22)$.

Spin saturation transfer experiments. Each of the four resonances belonging to the $\eta^{4}-\mathrm{C}_{6} \mathrm{H}_{8}$ ligand $(\delta 4.07,2.60,2.26$, and $1.64 \mathrm{ppm})$ was irradiated in a separate experiment. It was found that the irradiation of the resonance at $\delta 1.64 \mathrm{ppm}$ does not lead to a detectable change in the intensity of the other three. Irradiation of any of the remaining three resonances $(\delta 4.07,2.60$, $2.26 \mathrm{ppm}$ ) led to the decrease of the intensity of ca. $75 \%$ for the other two and had no effect on the intensity of resonance at $\delta 1.64 \mathrm{ppm}$.

Alkane activation by 3 at $165{ }^{\circ} \mathbf{C} . \quad 3(10 \mathrm{mg}, 15 \mu \mathrm{mol})$ was dissolved in $0.6 \mathrm{~mL}$ of ethylbenzene in a thick-walled flask equipped with a Teflon vacuum valve. This colorless solution was heated by means of an oil bath. No visible change occurred at temperatures below $150{ }^{\circ} \mathrm{C}$. The temperature was raised to $165{ }^{\circ} \mathrm{C}$ (red color of $\mathbf{1 2 h}$ began to develop) and the mixture was heated for $30 \mathrm{~min}$. Then the solution was cooled to $22{ }^{\circ} \mathrm{C}$ and analyzed by ${ }^{31} \mathrm{P}$ NMR. 12h was identified, corresponding to ca. $70 \%$ of combined intensity of the ${ }^{31} \mathrm{P}$ NMR 
resonances. Subsequent thermolysis at $165^{\circ} \mathrm{C}$ for $18 \mathrm{~h}$ resulted in a brown solution that no longer contained $\mathbf{1 2 h}$.

An analogous experiment was performed with $3(10 \mathrm{mg}, 15 \mu \mathrm{mol})$ dissolved in $0.6 \mathrm{~mL}$ of heptane. Upon raising the temperature above $150{ }^{\circ} \mathrm{C}$, the red color of $\mathbf{1 2 d}$ appeared, but after 18 $\mathrm{h}$ at $165^{\circ} \mathrm{C}$ the resultant brown solution no longer contained $\mathbf{1 2 d}$.

$\left(\mathrm{PNP}^{\mathrm{iPr}}\right) \operatorname{ReH}_{2}\left(=\mathrm{C}\left(\mathrm{CH}_{2}\right)_{4}\right) \quad(4 a), \quad\left(\mathrm{PNP}^{\mathrm{iPr}}\right) \operatorname{ReH}_{2}\left(\eta^{4}-\right.$ cyclopentadiene $) \quad(5 a)$, and $\quad\left(\kappa^{2}-P, P-\right.$ $\left.\mathbf{P N}(\mathbf{H}) \mathbf{P}^{\mathrm{iPr}}\right) \mathbf{R e H}_{2}\left(\eta^{5}-\mathbf{C p}\right)$ (6a). $\left(\mathrm{PNP}^{\mathrm{iPr}}\right) \mathrm{ReH}_{4}(\mathbf{1 a})(35 \mathrm{mg}, 60 \mu \mathrm{mol})$ was dissolved in $0.6 \mathrm{~mL}$ cyclo- $\mathrm{C}_{6} \mathrm{D}_{12}$ in a J. Young tube and cyclopentene $(21 \mu \mathrm{L}, 240 \mu \mathrm{mol})$ was added to it. After 10 min at $22{ }^{\circ} \mathrm{C},{ }^{31} \mathrm{P}$ NMR analysis indicated the formation of a mixture (slightly yellow) consisting of predominantly $\mathbf{4 a}, \mathbf{5 a}$, and $\mathbf{6 a}$ in ca. 10:10:1 ratio. After $5 \mathrm{~h}$ at $22{ }^{\circ} \mathrm{C}$, the signals of $4 \mathbf{a}$ completely disappeared and a mixture of $\mathbf{5 a}$ and $\mathbf{6 a}$ in 5:1 ratio was observed. The volatiles were removed in vacuo and the pale residue was redissolved in $\mathrm{C}_{6} \mathrm{D}_{6}$. This solution was heated at 90 ${ }^{\circ} \mathrm{C}$ for $3 \mathrm{~h}$. NMR analysis indicated $>95 \%$ conversion of $\mathbf{5 a}$ to $\mathbf{6 a}$. A solid sample of $\mathbf{6 a}$ was precipitated from a $\mathrm{SiMe}_{4}$ solution by addition of perfluorohexanes. NMR data follow. $\left(\mathbf{P N P}^{\mathrm{iPr}}\right) \operatorname{ReH}_{2}\left(=\mathbf{C}\left(\mathbf{C H}_{2}\right)_{4}\right)(\mathbf{4 a}) .{ }^{31} \mathrm{P}$ NMR $\left(\mathrm{C}_{6} \mathrm{D}_{12}\right): \delta 49.4$ (s). The hydride NMR signals are too broad to observe (cf those of $2 \mathbf{b}$ and 11a). (PNP $\left.{ }^{\text {iPr }}\right) \mathbf{R e H}_{2}\left(\eta^{4}\right.$-cyclopentadiene) (5a). ${ }^{1} \mathrm{H}$ NMR $\left(\mathrm{C}_{6} \mathrm{D}_{6}\right): \delta 5.67\left(\mathrm{dt}-\right.$ apparent q, $\left.1 \mathrm{H}, 9 \mathrm{~Hz}, \mathrm{HCH}(-\mathrm{CH}=\mathrm{CH})_{2}\right), 4.24\left(\mathrm{br} \mathrm{s}, 2 \mathrm{H}\right.$, pair of $\mathrm{C}\left(\mathrm{sp}^{2}\right)-\mathrm{H}$ of $\left.\mathrm{C}_{5} \mathrm{H}_{6}\right), 3.31$ (br d, $\left.1 \mathrm{H}, 9 \mathrm{~Hz}, \mathrm{HCH}(-\mathrm{CH}=\mathrm{CH})_{2}\right), 2.24\left(\mathrm{br} \mathrm{s}, 2 \mathrm{H}\right.$, pair of $\mathrm{C}\left(\mathrm{sp}^{2}\right)-H$ of $\left.\mathrm{C}_{5} \mathrm{H}_{6}\right), 2.11(\mathrm{br}$ m, 2H, PCH), 1.94 (br m, 2H, PCH), 1.19 (apparent q (dvt), 8 Hz, 6H, PCH-CH ) 1.09 (apparent q (dvt), $\left.8 \mathrm{~Hz}, 6 \mathrm{H}, \mathrm{PCH}-\mathrm{CH}_{3}\right), 1.06$ (apparent q (dvt), $8 \mathrm{~Hz}, 6 \mathrm{H}, \mathrm{PCH}^{-\mathrm{CH}_{3}}$ ), 0.97 (apparent q (dvt), $\left.8 \mathrm{~Hz}, 6 \mathrm{H}, \mathrm{PCH}-\mathrm{CH}_{3}\right), 0.29$ (s, 6H, SiMe), $0.18(\mathrm{~s}, 6 \mathrm{H}, \mathrm{SiMe}),-8.41\left(\mathrm{dt}, J_{\mathrm{HH}}=7 \mathrm{~Hz}, J_{\mathrm{HP}}=\right.$ $16 \mathrm{~Hz}, \mathrm{Re}-\mathrm{H})$; the $\mathrm{P}-\mathrm{CH}_{2}-\mathrm{Si}$ and the $2 \mathrm{nd} \mathrm{Re}-\mathrm{H}$ resonances are obscured by the aliphatic resonances. ${ }^{1} \mathrm{H}\left\{{ }^{31} \mathrm{P}\right\} \mathrm{NMR}\left(\mathrm{C}_{6} \mathrm{D}_{6}\right.$, selected resonances): $\delta 5.67\left(\mathrm{~d}, 1 \mathrm{H}, 9 \mathrm{~Hz}, \mathrm{HCH}(-\mathrm{CH}=\mathrm{CH})_{2}\right)$, 
4.24 (br s, $2 \mathrm{H}$, pair of $\mathrm{C}\left(\mathrm{sp}^{2}\right)-\mathrm{H}$ of $\left.\mathrm{C}_{5} \mathrm{H}_{6}\right), 3.31\left(\mathrm{~d}, 1 \mathrm{H}, 9 \mathrm{~Hz}, \mathrm{HCH}(-\mathrm{CH}=\mathrm{CH})_{2}\right), 2.24$ (br s, $2 \mathrm{H}$, pair of $\mathrm{C}\left(\mathrm{sp}^{2}\right)-H$ of $\left.\mathrm{C}_{5} \mathrm{H}_{6}\right),-8.41(\mathrm{~d}, 7 \mathrm{~Hz}, \mathrm{Re}-\mathrm{H}) .{ }^{13} \mathrm{C}\left\{{ }^{1} \mathrm{H}\right\}$ NMR $\left(\mathrm{C}_{6} \mathrm{D}_{6}\right): \delta 63.5\left(\mathrm{br}, \mathrm{C}\left(\mathrm{sp}^{2}\right) \mathrm{H}\right.$ of $\left.\mathrm{C}_{5} \mathrm{H}_{6}\right), 43.8\left(\mathrm{~s}, \mathrm{C}\left(\mathrm{sp}^{3}\right) \mathrm{H}_{2}\right.$ of $\left.\mathrm{C}_{5} \mathrm{H}_{6}\right), 31.2(\mathrm{~m}, \mathrm{PCH}), 26.0(\mathrm{~m}, \mathrm{PCH}), 25.2\left(\mathrm{br}, \mathrm{C}\left(\mathrm{sp}^{2}\right) \mathrm{H}_{\text {of }} \mathrm{C}_{5} \mathrm{H}_{6}\right)$, 21.1 (br s, $\mathrm{PCHMe}$ ), 20.6 (br s, $\mathrm{PCHMe}_{2}$ ), 19.6 (s, $\mathrm{PCHMe}_{2}$ ), 19.4 (s, $\mathrm{PCHMe}_{2}$ ), 19.2 (br s, $\left.\mathbf{P C H}_{2} \mathrm{Si}\right), \quad 6.6(\mathrm{~s}, \mathrm{SiMe}), 5.9$ (s, SiMe). ${ }^{31} \mathrm{P}\left\{{ }^{1} \mathrm{H}\right\} \quad \mathrm{NMR}\left(\mathrm{C}_{6} \mathrm{D}_{12}\right): \delta 28.2$ (br). $\quad\left(\boldsymbol{\kappa}^{2}-\boldsymbol{P}, \boldsymbol{P}-\right.$ $\left.\mathbf{P N}(\mathbf{H}) \mathbf{P}^{\mathrm{iPr}}\right) \mathbf{R e H}_{2}\left(\eta^{5}-\mathbf{C p}\right)$ (6a). ${ }^{1} \mathrm{H}$ NMR $\left(\mathrm{C}_{6} \mathrm{D}_{6}\right): \delta 4.57\left(\mathrm{~s}, 5 \mathrm{H}, \eta-\mathrm{C}_{5} \mathrm{H}_{5}\right), 1.73$ (m, 4H, PCH), $1.26\left(\mathrm{~d}, 8 \mathrm{~Hz}, \mathrm{PCH}_{2} \mathrm{Si}\right), 1.03$ (m, $24 \mathrm{H}, \mathrm{PCHMe}$ ), 0.24 (s, $\left.12 \mathrm{H}, \mathrm{SiMe}\right),-12.37$ (t, 2H, $41 \mathrm{~Hz}$, $\left.\mathrm{ReH}_{2}\right) .{ }^{13} \mathrm{C}\left\{{ }^{1} \mathrm{H}\right\}$ NMR $\left(\mathrm{C}_{6} \mathrm{D}_{6}\right): \delta 74.7\left(\eta-\mathrm{C}_{5} \mathrm{H}_{5}\right), 33.7$ (m, PCH), 23.4 (br s, $\left.\mathrm{PCH}_{2} \mathrm{Si}\right), 20.7$ (s, PCHMe 2 ), 19.6 (s, $\left.\mathrm{PCHMe}_{2}\right), 4.7$ (s, $\left.\mathrm{SiMe}_{3}\right) .{ }^{31} \mathrm{P}\left\{{ }^{1} \mathrm{H}\right\} \mathrm{NMR}\left(\mathrm{C}_{6} \mathrm{D}_{6}\right): \delta 26.4$ (s).

$\left(\mathbf{P N P}^{\mathrm{Cy}}\right) \operatorname{ReH}_{2}\left(=\mathbf{C}\left(\mathrm{CH}_{2}\right)_{4}\right) \mathbf{( 4 b )}$. 4b was observed as a minor component of the reactions of $\mathbf{1 b}$ with cyclopentene at $<30$ min reaction times. ${ }^{31} \mathrm{P}$ NMR $\left(\mathrm{C}_{6} \mathrm{D}_{6}\right): \delta 39.3$ (s).

(PNP $\left.{ }^{\mathrm{Cy}}\right) \mathbf{R e H}_{2}\left(\eta^{4}\right.$-cyclopentadiene) (5b). $\quad\left(\mathrm{PNP}^{\mathrm{Cy}}\right) \mathrm{ReH}_{4} \quad$ (1a) $(80 \mathrm{mg}, 103 \mu \mathrm{mol})$ was dissolved in $2 \mathrm{~mL}$ pentane and cyclopentene $(0.3 \mathrm{~mL}, 3.5 \mathrm{mmol})$ was added to it. The mixture was allowed to stand at $22{ }^{\circ} \mathrm{C}$ for $18 \mathrm{~h}$ and then was placed into a $-30{ }^{\circ} \mathrm{C}$ freezer overnight. The next day, the colorless crystals of $\mathbf{5 b}$ were collected by decantation and dried in vacuo to give 71 mg (85\%). ${ }^{1} \mathrm{H}$ NMR $\left(\mathrm{C}_{6} \mathrm{D}_{6}\right): \delta 5.72\left(\mathrm{dt}\right.$ - apparent q, $\left.1 \mathrm{H}, 9 \mathrm{~Hz}, \mathrm{HCH}(-\mathrm{CH}=\mathrm{CH})_{2}\right), 4.29(\mathrm{br} \mathrm{s}$, $2 \mathrm{H}$, pair of $\mathrm{C}\left(\mathrm{sp}^{2}\right)-\mathrm{H}$ of $\left.\mathrm{C}_{5} \mathrm{H}_{6}\right), 3.36\left(\right.$ br d, $\left.1 \mathrm{H}, 9 \mathrm{~Hz}, \mathrm{HCH}(-\mathrm{CH}=\mathrm{CH})_{2}\right), 2.29$ (br s, $2 \mathrm{H}$, pair of $\mathrm{C}\left(\mathrm{sp}^{2}\right)-\mathrm{H}$ of $\mathrm{C}_{5} \mathrm{H}_{6}$ ), 0.98-2.30 (several multiplets, $48 \mathrm{H}, \mathrm{PC}_{6} \mathrm{H}_{11}, \mathrm{PCH}_{2} \mathrm{Si}$ ), 0.35 (s, 6H, SiMe), $0.27(\mathrm{~s}, 6 \mathrm{H}, \mathrm{SiMe}),-8.04\left(\mathrm{dt}, J_{\mathrm{HH}}=7 \mathrm{~Hz}, J_{\mathrm{HP}}=16 \mathrm{~Hz}, \mathrm{Re}-H\right) .{ }^{31} \mathrm{P}\left\{{ }^{1} \mathrm{H}\right\} \operatorname{NMR}\left(\mathrm{C}_{6} \mathrm{D}_{12}\right): \delta 17.7$ (br).

Thermolysis of $\mathbf{5 b}$. $5 \mathbf{b}(17 \mathrm{mg})$ was dissolved in $0.6 \mathrm{~mL} \mathrm{C}_{6} \mathrm{D}_{6}$ and heated at $90{ }^{\circ} \mathrm{C}$ for $1 \mathrm{~h}$. NMR analysis indicated the formation of $\mathbf{1 3}$ (H/D isotopomers) and $\mathbf{6 b}$ in a 54:46 ratio. Further heating did not change this ratio. 
$\left(\mathbf{P N P}{ }^{\mathrm{iPr}}\right) \mathbf{R e H}\left(\equiv \mathbf{C}-\left(\mathbf{C H}_{2}\right)_{3} \mathbf{C H}_{3}\right)$ (12b). $\left(\mathrm{PNP}^{\mathrm{iPr}}\right) \mathrm{ReH}_{2}$ (cyclooctyne) $(\mathbf{8})(25 \mathrm{mg}, 36 \mu \mathrm{mol})$ was dissolved in a mixture of $0.3 \mathrm{~mL}$ cyclooctane and $0.3 \mathrm{~mL}$ pentane in a J. Young tube. The solution was degassed by two freeze-pump-thaw cycles and then the tube was inserted into a 110 ${ }^{\circ} \mathrm{C}$ oil bath. After 24 hours the conversion is essentially quantitative by NMR. The volatiles were removed from the solution in vacuo, leaving behind a viscous red oil. It was redissolved in $\mathrm{C}_{6} \mathrm{D}_{6}$ for full NMR characterization.

${ }^{1} \mathrm{H}$ NMR $\left(\mathrm{C}_{6} \mathrm{D}_{6}\right): \delta 2.14\left(\mathrm{~m}, \mathrm{~J}_{\mathrm{HH}}=7 \mathrm{~Hz}, 2 \mathrm{H}, \mathrm{PCH}\right), 1.79\left(\mathrm{~m}, \mathrm{~J}_{\mathrm{HH}}=7 \mathrm{~Hz}, 2 \mathrm{H}, \mathrm{PCH}\right), 1.65(\mathrm{tt}$, $\left.\mathrm{J}_{\mathrm{HH}}=7 \mathrm{~Hz}, 2 \mathrm{H}, \mathrm{Re} \equiv \mathrm{C}-\mathrm{CH}_{2}\right), 1.51\left(\mathrm{tt}, 2 \mathrm{H}, \mathrm{Re} \equiv \mathrm{C}-\mathrm{CH}_{2}-\mathrm{CH}_{2}\right), 1.38\left(\mathrm{tq}, 2 \mathrm{H}, \mathrm{Re} \equiv \mathrm{C}-\mathrm{CH}_{2}-\mathrm{CH}_{2}-\mathrm{CH}_{2}\right)$, 1.29 (apparent q (dvt), $\left.8 \mathrm{~Hz}, 6 \mathrm{H}, \mathrm{PCH}-\mathrm{CH}_{3}\right), 1.19$ (apparent q (dvt), $\left.8 \mathrm{~Hz}, 6 \mathrm{H}, \mathrm{PCH}-\mathrm{CH}_{3}\right), 1.07$ (apparent q (dvt), $8 \mathrm{~Hz}, 6 \mathrm{H}, \mathrm{PCH}-\mathrm{CH}_{3}$ ), 1.05 (apparent q (dvt), $8 \mathrm{~Hz}, 6 \mathrm{H}, \mathrm{PCH}-\mathrm{CH}_{3}$ ), 0.85 (t, 7 $\left.\mathrm{Hz}, 3 \mathrm{H}, \mathrm{Re} \equiv \mathrm{C}-\mathrm{CH}_{2}-\mathrm{CH}_{2}-\mathrm{CH}_{2}-\mathrm{CH}_{3}\right), 0.83\left(\mathrm{AB} \mathrm{dvt}, \mathrm{J}_{\mathrm{HH}}=14 \mathrm{~Hz}, \mathrm{~J}_{\mathrm{HP}}=4 \mathrm{~Hz}, 2 \mathrm{H}, \mathrm{PCH}_{2} \mathrm{Si}\right), 0.75$ $\left(\mathrm{AB} d v t, \mathrm{~J}_{\mathrm{HH}}=14 \mathrm{~Hz}, \mathrm{~J}_{\mathrm{HP}}=4 \mathrm{~Hz}, 2 \mathrm{H}, \mathrm{PCH}_{2} \mathrm{Si}\right), 0.37\left(\mathrm{~s}, 6 \mathrm{H}, \mathrm{SiCH}_{3}\right), 0.32\left(\mathrm{~s}, 6 \mathrm{H}, \mathrm{SiCH}_{3}\right),-10.04$ (t, $14 \mathrm{~Hz}, 1 \mathrm{H}, \mathrm{Re} H) .{ }^{31} \mathrm{P}\left\{{ }^{1} \mathrm{H}\right\}$ NMR $\left(\mathrm{C}_{6} \mathrm{D}_{6}\right): \delta 61.03(\mathrm{~s}) .{ }^{13} \mathrm{C}\left\{{ }^{1} \mathrm{H}\right\} \mathrm{NMR}\left(\mathrm{C}_{6} \mathrm{D}_{6}\right): \delta 279.3(\mathrm{t}, 11 \mathrm{~Hz}$, $\mathrm{Re} \equiv C), 50.9\left(\mathrm{~s}, \mathrm{Re} \equiv \mathrm{C}-\mathrm{CH}_{2}\right), 29.5(\mathrm{t}, 13 \mathrm{~Hz}, \mathrm{P}-\mathrm{CH}), 29.2(\mathrm{t}, 11 \mathrm{~Hz}, \mathrm{P}-\mathrm{CH}), 27.0\left(\mathrm{~s}, \mathrm{Re}=\mathrm{C}-\mathrm{CH}_{2^{-}}\right.$ $\left.\mathrm{CH}_{2}\right), 22.2\left(\mathrm{~s}, \mathrm{Re} \equiv \mathrm{C}-\mathrm{CH}_{2}-\mathrm{CH}_{2}-\mathrm{CH}_{2}\right), 20.3\left(\mathrm{~s}, \mathrm{PCH}-\mathrm{CH}_{3}\right), 19.0\left(\mathrm{~s}, \mathrm{PCH}-\mathrm{CH}_{3}\right), 18.8$ (s, PCH$\left.\mathrm{CH}_{3}\right), 17.3$ (s, PCH-CH$)_{3}, 14.1$ (s, $\left.\mathrm{Re} \equiv \mathrm{C}-\mathrm{CH}_{2}-\mathrm{CH}_{2}-\mathrm{CH}_{2}-\mathrm{CH}_{3}\right), 10.6(\mathrm{~s}, \mathrm{PCH} 2 \mathrm{Si}), 7.3\left(\mathrm{~s}, \mathrm{SiCH}_{3}\right)$, $6.3\left(\right.$ br s, $\left.\mathrm{SiCH}_{3}\right)$.

$\left(\mathbf{P N P}^{\mathrm{iPr}}\right) \operatorname{ReH}\left(\equiv \mathbf{C}-\left(\mathbf{C H}_{2}\right)_{4} \mathbf{C H}_{3}\right)$ (12c). $\left(\mathrm{PNP}^{\mathrm{iPr}}\right) \mathrm{ReH}_{2}$ (cyclooctyne) (8) $(25 \mathrm{mg}, 36 \mu \mathrm{mol})$ was dissolved in $0.6 \mathrm{~mL}$ hexane in a J. Young tube. The tube was inserted into a $110{ }^{\circ} \mathrm{C}$ oil bath. After 24 hours the conversion is essentially quantitative by NMR. The volatiles were removed from the solution in vacuo, leaving behind a viscous red oil. It was redissolved in $\mathrm{C}_{6} \mathrm{D}_{6}$ for full NMR characterization. The NMR data were identical to those reported previously for this compound. 
$\left(\mathbf{P N P}{ }^{\mathrm{iPr}}\right) \mathbf{R e H}\left(\equiv \mathbf{C}-\left(\mathbf{C H}_{2}\right)_{5} \mathbf{C H}_{3}\right)$ (12d). $\left(\mathrm{PNP}^{\mathrm{iPr}}\right) \mathrm{ReH}_{2}$ (cyclooctyne) $(\mathbf{8})(25 \mathrm{mg}, 36 \mu \mathrm{mol})$ was dissolved in $0.6 \mathrm{~mL}$ heptane in a $\mathrm{J}$. Young tube. The tube was inserted into a $110{ }^{\circ} \mathrm{C}$ oil bath. After 24 hours the conversion is essentially quantitative by NMR. The volatiles were removed from the solution in vacuo, leaving behind a viscous red oil. It was redissolved in $\mathrm{C}_{6} \mathrm{D}_{6}$ for full NMR characterization.

${ }^{1} \mathrm{H}$ NMR $\left(\mathrm{C}_{6} \mathrm{D}_{6}\right): \delta 2.15\left(\mathrm{~m}, \mathrm{~J}_{\mathrm{HH}}=7 \mathrm{~Hz}, 2 \mathrm{H}, \mathrm{PCH}\right), 1.80\left(\mathrm{~m}, \mathrm{~J}_{\mathrm{HH}}=7 \mathrm{~Hz}, 2 \mathrm{H}, \mathrm{PCH}\right), 1.67(\mathrm{tt}$, $\left.\mathrm{J}_{\mathrm{HH}}=7 \mathrm{~Hz}, 2 \mathrm{H}, \mathrm{Re} \equiv \mathrm{C}-\mathrm{CH}_{2}\right), 1.55\left(\mathrm{tt}, 2 \mathrm{H}, \mathrm{Re} \equiv \mathrm{C}-\mathrm{CH}_{2}-\mathrm{CH}_{2}\right), 1.37$ (tt, $\left.2 \mathrm{H}, \mathrm{Re} \equiv \mathrm{C}-\mathrm{CH}_{2}-\mathrm{CH}_{2}-\mathrm{CH}_{2}\right)$, 1.30 (apparent q (dvt), $\left.8 \mathrm{~Hz}, 6 \mathrm{H}, \mathrm{PCH}-\mathrm{CH}_{3}\right), 1.20-1.25$ (br, 4H, $2 \mathrm{CH}_{2}$ 's of heptylidyne), 1.21 (apparent q (dvt), $8 \mathrm{~Hz}, 6 \mathrm{H}, \mathrm{PCH}-\mathrm{CH}_{3}$ ), 1.08 (apparent q (dvt), $8 \mathrm{~Hz}, 6 \mathrm{H}, \mathrm{PCH}-\mathrm{CH}_{3}$ ), 1.05 (apparent q (dvt), $\left.8 \mathrm{~Hz}, 6 \mathrm{H}, \mathrm{PCH}-\mathrm{CH}_{3}\right), 0.88\left(\mathrm{t}, 7 \mathrm{~Hz}, 3 \mathrm{H}, \mathrm{Re} \equiv \mathrm{C}-\left(\mathrm{CH}_{2}\right)_{6} \mathrm{CH}_{3}\right), 0.84\left(\mathrm{AB} \mathrm{dvt}, \mathrm{J}_{\mathrm{HH}}\right.$ $\left.\left.=14 \mathrm{~Hz}, \mathrm{~J}_{\mathrm{HP}}=4 \mathrm{~Hz}, 2 \mathrm{H}, \mathrm{PCH}_{2} \mathrm{Si}\right),\right), 0.75\left(\mathrm{AB} \mathrm{dvt}, \mathrm{J}_{\mathrm{HH}}=14 \mathrm{~Hz}, \mathrm{~J}_{\mathrm{HP}}=4 \mathrm{~Hz}, 2 \mathrm{H}, \mathrm{PCH}_{2} \mathrm{Si}\right), 0.38$ (s, 6H, $\left.\mathrm{SiCH}_{3}\right), 0.32\left(\mathrm{~s}, 6 \mathrm{H}, \mathrm{SiCH}_{3}\right),-10.02(\mathrm{t}, 14 \mathrm{~Hz}, 1 \mathrm{H}, \mathrm{Re} H) .{ }^{31} \mathrm{P}\left\{{ }^{1} \mathrm{H}\right\} \mathrm{NMR}\left(\mathrm{C}_{6} \mathrm{D}_{6}\right): \delta 61.00$ (s). ${ }^{13} \mathrm{C}\left\{{ }^{1} \mathrm{H}\right\}$ NMR $\left(\mathrm{C}_{6} \mathrm{D}_{6}\right): \delta 279.4(\mathrm{t}, 11 \mathrm{~Hz}, \mathrm{Re} \equiv \mathrm{C}), 51.2\left(\mathrm{~s}, \mathrm{Re} \equiv \mathrm{C}-\mathrm{CH}_{2}\right), 32.0\left(\mathrm{~s}, \mathrm{CH}_{2}\right.$ of heptylidyne), 29.5 (t, $13 \mathrm{~Hz}, \mathrm{P}-\mathrm{CH} 29.2$ (t, $11 \mathrm{~Hz}, \mathrm{P}-\mathrm{CH}$ ), 28.9 (s, $\mathrm{CH}_{2}$ of heptylidyne), 27.4 (s, $\mathrm{CH}_{2}$ of heptylidyne), 23.0 (s, $\mathrm{CH}_{2}$ of heptylidyne), 20.3 (t, $2 \mathrm{~Hz}, \mathrm{PCH}_{-} \mathrm{CH}_{3}$ ), 19.0 (br s, $\mathrm{PCH}-$ $\mathrm{CH}_{3}$ ), 18.8 (br s, PCH-CH 3 ), 17.3 (br s, $\left.\mathrm{PCH}-\mathrm{CH}_{3}\right), 14.3$ (s, $\left.\mathrm{Re}=\mathrm{C}-\left(\mathrm{CH}_{2}\right)_{5}-\mathrm{CH}_{3}\right), 10.6$ (s, $\left.\mathrm{PCH}_{2} \mathrm{Si}\right), 7.3$ (s, $\left.\mathrm{SiCH}_{3}\right), 6.3\left(\mathrm{t}, 3 \mathrm{~Hz}, \mathrm{SiCH}_{3}\right)$.

$\left(\mathbf{P N P}^{\mathrm{iPr}}\right) \mathbf{R e H}\left(\equiv \mathbf{C}-\left(\mathbf{C H}_{2}\right)_{6} \mathbf{C H}_{3}\right)(\mathbf{1 2 e}) .\left(\mathrm{PNP}^{\mathrm{iPr}}\right) \mathrm{ReH}_{2}$ (cyclooctyne) $(\mathbf{8})(25 \mathrm{mg}, 36 \mu \mathrm{mol})$ was dissolved in $0.6 \mathrm{~mL}$ octane in a J. Young tube. The tube was inserted into a $110{ }^{\circ} \mathrm{C}$ oil bath. After 24 hours the conversion is essentially quantitative by NMR. The volatiles were removed from the solution in vacuo, leaving behind a viscous red oil. It was redissolved in $\mathrm{C}_{6} \mathrm{D}_{6}$ for full NMR characterization. 
${ }^{1} \mathrm{H}$ NMR $\left(\mathrm{C}_{6} \mathrm{D}_{6}\right): \delta 2.16\left(\mathrm{~m}, \mathrm{~J}_{\mathrm{HH}}=7 \mathrm{~Hz}, 2 \mathrm{H}, \mathrm{PCH}\right), 1.80\left(\mathrm{~m}, \mathrm{~J}_{\mathrm{HH}}=7 \mathrm{~Hz}, 2 \mathrm{H}, \mathrm{PCH}\right), 1.67(\mathrm{tt}$, $\left.\mathrm{J}_{\mathrm{HH}}=7 \mathrm{~Hz}, 2 \mathrm{H}, \mathrm{Re} \equiv \mathrm{C}-\mathrm{CH}_{2}\right), 1.56\left(\mathrm{tt}, 2 \mathrm{H}, \mathrm{Re} \equiv \mathrm{C}-\mathrm{CH}_{2}-\mathrm{CH}_{2}\right), 1.38\left(\mathrm{tt}, 2 \mathrm{H}, \mathrm{Re} \equiv \mathrm{C}-\mathrm{CH}_{2}-\mathrm{CH}_{2}-\mathrm{CH}_{2}\right)$, 1.30 (apparent q (dvt), $\left.8 \mathrm{~Hz}, 6 \mathrm{H}, \mathrm{PCH}-\mathrm{CH}_{3}\right), 1.24$ (br, 6H,3 $\mathrm{CH}_{2}$ 's of octylidyne), 1.21 (apparent q (dvt), $\left.8 \mathrm{~Hz}, 6 \mathrm{H}, \mathrm{PCH}-\mathrm{CH}_{3}\right), 1.08$ (apparent q (dvt), $8 \mathrm{~Hz}, 6 \mathrm{H}, \mathrm{PCH}-\mathrm{CH}_{3}$ ), 1.05 (apparent q (dvt), $\left.8 \mathrm{~Hz}, 6 \mathrm{H}, \mathrm{PCH}-\mathrm{CH}_{3}\right), 0.88\left(\mathrm{t}, 7 \mathrm{~Hz}, 3 \mathrm{H}, \mathrm{Re}=\mathrm{C}-\left(\mathrm{CH}_{2}\right)_{6} \mathrm{CH}_{3}\right), 0.84\left(\mathrm{AB} \mathrm{dvt}, \mathrm{J}_{\mathrm{HH}}=14 \mathrm{~Hz}, \mathrm{~J}_{\mathrm{HP}}\right.$ $\left.=4 \mathrm{~Hz}, 2 \mathrm{H}, \mathrm{PCH}_{2} \mathrm{Si}\right)$, ), $0.75\left(\mathrm{AB} \mathrm{dvt}, \mathrm{J}_{\mathrm{HH}}=14 \mathrm{~Hz}, \mathrm{~J}_{\mathrm{HP}}=4 \mathrm{~Hz}, 2 \mathrm{H}, \mathrm{PCH}_{2} \mathrm{Si}\right), 0.38(\mathrm{~s}, 6 \mathrm{H}$, $\left.\mathrm{SiCH}_{3}\right), 0.32\left(\mathrm{~s}, 6 \mathrm{H}, \mathrm{SiCH}_{3}\right),-10.02(\mathrm{t}, 14 \mathrm{~Hz}, 1 \mathrm{H}, \mathrm{ReH}) .{ }^{31} \mathrm{P}\left\{{ }^{1} \mathrm{H}\right\}$ NMR $\left(\mathrm{C}_{6} \mathrm{D}_{6}\right): \delta 61.02(\mathrm{~s})$. ${ }^{13} \mathrm{C}\left\{{ }^{1} \mathrm{H}\right\}$ NMR $\left(\mathrm{C}_{6} \mathrm{D}_{6}\right): \delta 279.4(\mathrm{t}, 11 \mathrm{~Hz}, \mathrm{Re} \equiv \mathrm{C}), 51.2\left(\mathrm{~s}, \mathrm{Re} \equiv \mathrm{C}-\mathrm{CH}_{2}\right), 32.2\left(\mathrm{~s}, \mathrm{CH}_{2}\right.$ of octylidyne), 29.50 (s, $\mathrm{CH}_{2}$ of octylidyne), 29.48 (t, $13 \mathrm{~Hz}, \mathrm{P}-\mathrm{CH}$ ), 29.23 (s, $\mathrm{CH}_{2}$ of octylidyne), 29.17 (t, $11 \mathrm{~Hz}, \mathrm{P}-\mathrm{CH}$ ), 27.4 (s, $\mathrm{CH}_{2}$ of octylidyne), 23.0 (s, $\mathrm{CH}_{2}$ of octylidyne), 20.3 (s, PCH$\left.\mathrm{CH}_{3}\right), 19.1$ (s, PCH-CH 3$), 18.8\left(\mathrm{~s}, \mathrm{PCH}-\mathrm{CH}_{3}\right), 17.3\left(\mathrm{~s}, \mathrm{PCH}-\mathrm{CH}_{3}\right), 14.3\left(\mathrm{~s}, \mathrm{Re} \equiv \mathrm{C}-\left(\mathrm{CH}_{2}\right)_{6}-\mathrm{CH}_{3}\right)$, $10.6\left(\mathrm{~s}, \mathrm{PCH}_{2} \mathrm{Si}\right), 7.3\left(\mathrm{~s}, \mathrm{SiCH}_{3}\right), 6.3\left(\right.$ br s, $\left.\mathrm{SiCH}_{3}\right)$.

$\left(\mathbf{P N P}{ }^{\mathrm{iPr}}\right) \mathbf{R e H}\left(\equiv \mathbf{C}-\mathbf{C H}_{2}-\mathbf{C H}\left(\mathbf{C H}_{3}\right)-\mathbf{C H}_{2}-\mathbf{C H}_{3}\right)(\mathbf{1 2 f}) .\left(\mathrm{PNP}^{\mathrm{iPr}}\right) \mathrm{ReH}_{4}(\mathbf{1 a})(26 \mathrm{mg}, 45 \mu \mathrm{mol})$ was dissolved in $0.6 \mathrm{~mL}$ 3-methylpentane in a J. Young tube and cyclooctene $(20.0 \mu \mathrm{L}, 154 \mu \mathrm{mol})$ was added to it. The solution was degassed by two freeze-pump-thaw cycles and then the tube was inserted into a $110^{\circ} \mathrm{C}$ oil bath. After $1.5 \mathrm{~h}$ the product $\mathbf{1 2 f}$ and $\mathbf{8}$ were observed in ca. 40:60 ratio by ${ }^{31} \mathrm{P}$ NMR. After $18 \mathrm{~h}$, the conversion to $\mathbf{1 2 f}$ was $>95 \%$ by ${ }^{31} \mathrm{P}$ NMR. The volatiles were removed from the solution in vacuo, leaving behind a viscous red oil. It was redissolved in $\mathrm{C}_{6} \mathrm{D}_{6}$ for NMR characterization.

${ }^{1} \mathrm{H} \operatorname{NMR}\left(\mathrm{C}_{6} \mathrm{D}_{6}\right): \delta 2.16\left(\mathrm{~m}, \mathrm{~J}_{\mathrm{HH}}=7 \mathrm{~Hz}, 2 \mathrm{H}, \mathrm{PCH}\right), 1.78\left(\mathrm{~m}, \mathrm{~J}_{\mathrm{HH}}=7 \mathrm{~Hz}, 2 \mathrm{H}, \mathrm{PCH}\right), 1.67(\mathrm{~m}$, 2H, Re $\left.\equiv \mathrm{C}-\mathrm{CH}_{2}\right), 1.57\left(\mathrm{~m}, 1 \mathrm{H}, \mathrm{Re} \equiv \mathrm{C}-\mathrm{CH}_{2}-\mathrm{CH}\right), 1.43\left(\mathrm{~m}, 2 \mathrm{H}, \mathrm{Re} \equiv \mathrm{C}-\mathrm{CH}_{2}-\mathrm{CH}\left(\mathrm{CH}_{3}\right)-\mathrm{CH}_{2}\right), 1.29$ (apparent q (dvt), $8 \mathrm{~Hz}, 6 \mathrm{H}, \mathrm{PCH}-\mathrm{CH}_{3}$ ), 1.22 (apparent q (dvt), $8 \mathrm{~Hz}, 6 \mathrm{H}, \mathrm{PCH}-\mathrm{CH}_{3}$ ), 1.06 (apparent q (dvt), $\left.8 \mathrm{~Hz}, 6 \mathrm{H}, \mathrm{PCH}-\mathrm{CH}_{3}\right), 1.04$ (apparent q (dvt), $\left.8 \mathrm{~Hz}, 6 \mathrm{H}, \mathrm{PCH}-\mathrm{CH}_{3}\right), 1.01$ (d, 8 
$\left.\mathrm{Hz}, 3 \mathrm{H}, \mathrm{Re} \equiv \mathrm{C}-\mathrm{CH}_{2}-\mathrm{CH}\left(\mathrm{CH}_{3}\right)-\mathrm{CH}_{2} \mathrm{CH}_{3}\right), 0.85\left(\mathrm{t}, 8 \mathrm{~Hz}, 3 \mathrm{H}, \mathrm{Re} \equiv \mathrm{C}-\mathrm{CH}_{2}-\mathrm{CH}\left(\mathrm{CH}_{3}\right)-\mathrm{CH}_{2}-\mathrm{CH}_{3}\right), 0.82$ $\left(\mathrm{AB} d v t, \mathrm{~J}_{\mathrm{HH}}=14 \mathrm{~Hz}, \mathrm{~J}_{\mathrm{HP}}=4 \mathrm{~Hz}, 2 \mathrm{H}, \mathrm{PCH}_{2} \mathrm{Si}\right), 0.74\left(\mathrm{AB} \mathrm{dvt}, \mathrm{J}_{\mathrm{HH}}=14 \mathrm{~Hz}, \mathrm{~J}_{\mathrm{HP}}=4 \mathrm{~Hz}, 2 \mathrm{H}\right.$, $\left.\mathrm{PCH}_{2} \mathrm{Si}\right), 0.38\left(\mathrm{~s}, 6 \mathrm{H}, \mathrm{SiCH}_{3}\right), 0.32\left(\mathrm{~s}, 6 \mathrm{H}, \mathrm{SiCH}_{3}\right),-9.98(\mathrm{t}, 14 \mathrm{~Hz}, 1 \mathrm{H}, \mathrm{ReH}) .{ }^{31} \mathrm{P}\left\{{ }^{1} \mathrm{H}\right\} \mathrm{NMR}$ $\left(\mathrm{C}_{6} \mathrm{D}_{6}\right): \delta 60.40(\mathrm{~s})$

$\left.\left(\mathbf{P N P}{ }^{\mathrm{iPr}}\right) \mathbf{R e H}\left(\equiv \mathbf{C}-\mathbf{C H}_{2} \mathbf{C}\left(\mathbf{C H}_{\mathbf{3}}\right)_{3}\right) \mathbf{( 1 2 g}\right) .\left(\mathrm{PNP}^{\mathrm{iPr}}\right) \mathrm{ReH}_{2}$ (cyclooctyne) (8) (25 mg, $\left.36 \mu \mathrm{mol}\right)$ was dissolved in $0.6 \mathrm{~mL}$ neo-hexane in a J. Young tube. The tube was inserted into a $110{ }^{\circ} \mathrm{C}$ oil bath. After 48 hours 8 was consumed and $\mathbf{1 2 g}$ corresponded to ca. $75 \%$ of the mixture by ${ }^{31} \mathrm{P}$ NMR. The NMR data were identical to those reported previously\# for this compound.

Competition experiments. Equimolar amounts of two substrates were mixed to a total volume of $0.600 \mathrm{~mL}$ and $\left(\mathrm{PNP}^{\mathrm{iPr}}\right) \mathrm{ReH}_{2}$ (cyclooctyne) $(\mathbf{8})(10 \mathrm{mg}, 15 \mu \mathrm{mol})$ was added to this mixture. The mixture was thermolyzed at $110^{\circ} \mathrm{C}$ and monitored by ${ }^{31} \mathrm{P}$ NMR. In all cases, after $18 \mathrm{~h}$, the consumption of $\mathbf{8}$ was complete.

a) Hexane $(350 \mu \mathrm{L})+$ cyclopentane $(250 \mu \mathrm{L}), 2.68 \mathrm{mmol}$ each. Final ratio of $\mathbf{1 2 c}$ to $\mathbf{6 a}-$ 73:27.

b) Heptane $(366 \mu \mathrm{L})+$ cyclopentane $(234 \mu \mathrm{L}), 2.50 \mathrm{mmol}$ each. Final ratio of $\mathbf{1 2 d}$ to $\mathbf{6 a}-$ 73:27.

c) Hexane $(310 \mu \mathrm{L})+$ ethylbenzene $(290 \mu \mathrm{L}), 2.37 \mathrm{mmol}$ each. Final ratio of $\mathbf{1 2 c}$ to $\mathbf{1 2 h}-$ $\underline{52: 48}$.

d) Heptane $(327 \mu \mathrm{L})+$ ethylbenzene $(273 \mu \mathrm{L}), 2.23 \mathrm{mmol}$ each. Final ratio of $\mathbf{1 2 d}$ to $\mathbf{1 2 h}-$ $\underline{53: 47}$.

Cyclometallated compound 13. $\left(\mathrm{PNP}^{\mathrm{iPr}}\right) \mathrm{Re}(\mathrm{O}) \mathrm{Cl}_{2}(2.5 \mathrm{~g}, 2.9 \mathrm{mmol})$ was dissolved in $10 \mathrm{~mL}$ of $\mathrm{Et}_{2} \mathrm{O}$ in a screw-cap tube with a spin bar. $\mathrm{Mg}$ powder (ca. $0.5 \mathrm{~g}, 20 \mathrm{mmol}$ ) was added to it, the cap was closed and the mixture was stirred vigorously for $24 \mathrm{~h}$. During this time the color 
changed to purple. ${ }^{31} \mathrm{P}$ NMR analysis indicated a 10:1 mixture of $\mathbf{1 3}$ to $\mathbf{1 b}$ (this and similar experiments have produced ratios of 3:1 to 10:1, apparently depending on the quality of the $\mathrm{Mg}$ ). The solution was treated with $1 \mathrm{~mL}$ dioxane, and the volatiles were removed in vacuo. The residue was extracted with ether and filtered and the volatiles were removed from the filtrate in vacuo. The residue was recrystallized from ether/pentane at $-30{ }^{\circ} \mathrm{C}$ to give $1.31 \mathrm{~g}(59 \%)$ of $\mathbf{1 3}$.

${ }^{1} \mathrm{H}$ NMR $\left(\mathrm{C}_{6} \mathrm{D}_{6}\right): \delta 4.56(\mathrm{~m}, 1 \mathrm{H}, \mathrm{CH}$ of P-cyclohexenyl), 3.33 (br s, $1 \mathrm{H}, \mathrm{CH}$ of Pcyclohexenyl), 3.13 (br s, 1H, CH of P-cyclohexenyl), 2.16-2.38, 1.48-2.00, 1.36-1.46, 0.981.30 (several multiplets, 39H, P-cyclohexyl, P-cyclohexenyl, $\mathrm{PCH}_{2} \mathrm{Si}$ ), 0.82 (m, 1H, $\mathrm{PCH}_{2} \mathrm{Si}$ ), 0.62 (s, 3H, SiMe), 0.53 (s, 3H, SiMe), 0.39-0.52 (m, 2H, $\mathrm{PCH}_{2} \mathrm{Si}$ ), 0.26 (s, 3H, SiMe), 0.25 (s, 3H, SiMe), $-9.63(\mathrm{dd}, 18 \mathrm{~Hz}, 8 \mathrm{~Hz}, \mathrm{Re}-H) .{ }^{31} \mathrm{P}\left\{{ }^{1} \mathrm{H}\right\}$ NMR $\left(\mathrm{C}_{6} \mathrm{D}_{12}\right): \delta 85.3(\mathrm{~d}, 239 \mathrm{~Hz}), 34.9(\mathrm{~d}$, $239 \mathrm{~Hz}) .{ }^{13} \mathrm{C}\left\{{ }^{1} \mathrm{H}\right\}$ NMR $\left(\mathrm{C}_{6} \mathrm{D}_{6}\right): \delta 61.6$ (s, CH of P-cyclohexenyl), 52.3 (s, CH of Pcyclohexenyl), 47.9 (s, CH of P-cyclohexenyl), 42.4 (d, $21 \mathrm{~Hz}), 39.9$ (d, $25 \mathrm{~Hz}), 39.6$ (d, $22 \mathrm{~Hz})$, 36.2 (d, $14 \mathrm{~Hz}$ ), 34.0 (d, $14 \mathrm{~Hz}), 31.3$ (d, $11 \mathrm{~Hz}), 30.1$ (d, $4 \mathrm{~Hz}), 28.9$ (d, $3 \mathrm{~Hz}), 28.8$ (dd, $3 \mathrm{~Hz}, 2$ Hz), 28.7 (br s), 28.5 (d, 3 Hz), 27.9 (s), 27.8 (br s), 27.7 (d, 4 Hz), 27.6 (s), 27.5 (s), 27.4 (s), 27.3 (d, 4 Hz), 27.2 (br s), 27.03 (d, $6 \mathrm{~Hz}$ ), 26.98 (br s), 10.1 (br d, $\mathrm{PCH}_{2} \mathrm{Si}$ ), 9.8 (s, $\mathrm{PCH}_{2} \mathrm{Si}$ ), 8.8 (d, 3 Hz, SiMe), 7.6 (d, 5 Hz, SiMe), 5.8 (d, 3 Hz, SiMe), 4.6 (d, 5 Hz, SiMe). 
Pnpreh4

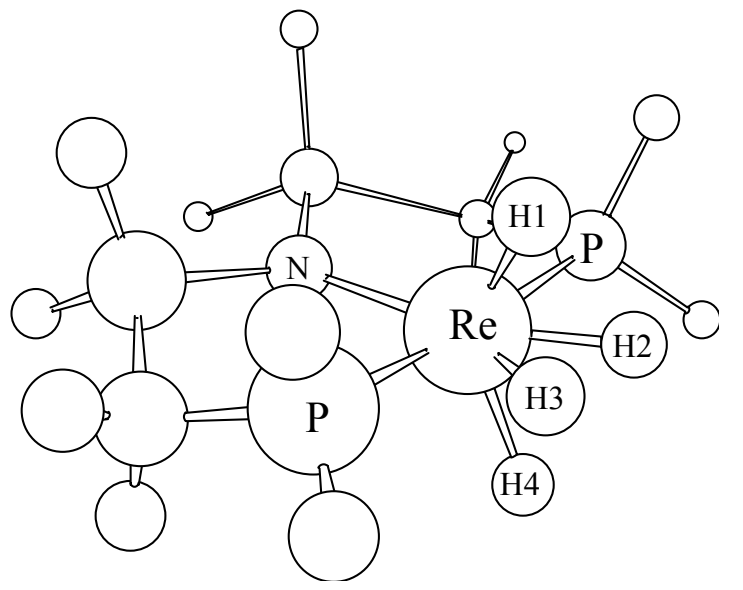

Selected Bond Distances $(\AA)$ and Angles $\left({ }^{\circ}\right)$ : Re-N(PNP): 2.062

Re-H1: 1.639

Re-H2: 1.664

Re-H3: 1.664

Re-H4: 1.645

H3-H4: 1.525

N-Si: 1.761

Re-P: 2.374

$\angle \mathrm{N}-\mathrm{Re}-\mathrm{H} 1: 114.3$

$\angle \mathrm{N}-\mathrm{Re}-\mathrm{H} 4: 118.0$

\footnotetext{
Atomic Coordinates (XYZ)

Re $0.00013-0.861220 .03245$

P $2.36656-0.67382-0.00161$

C $2.881891 .03894-0.48524$

Si 1.484952 .140310 .13968

H 1.778022 .558631 .53736

H $1.400913 .37830-0.68009$

N $-0.00021 \quad 1.201240 .02649$

Si -1.48539 $2.14058 \quad 0.13791$

$\mathrm{H}-1.401303 .37676-0.68455$

$\mathrm{H}-1.778122 .56194 \quad 1.53475$

P - $2.36637-0.67436-0.00078$

C $-2.88251 \quad 1.03812-0.48475$

H $3.871841 .30981-0.11006$

H $2.894261 .08136-1.57927$

H -2.89642 $1.08009-1.57878$

H -3.87215 $1.30858-0.10845$

H $3.10685-0.87521 \quad 1.20257$

H $3.14767-1.52317-0.83675$

H -3.14741 -1.52416 - 0.83551

H -3.10635 $-0.87561 \quad 1.20362$

H $0.00019-1.529921 .52839$

H - $0.76182-2.33920 \quad 0.08747$

H - $0.00016-1.63864-1.41692$

H $0.76306-2.338670 .08665$
} 


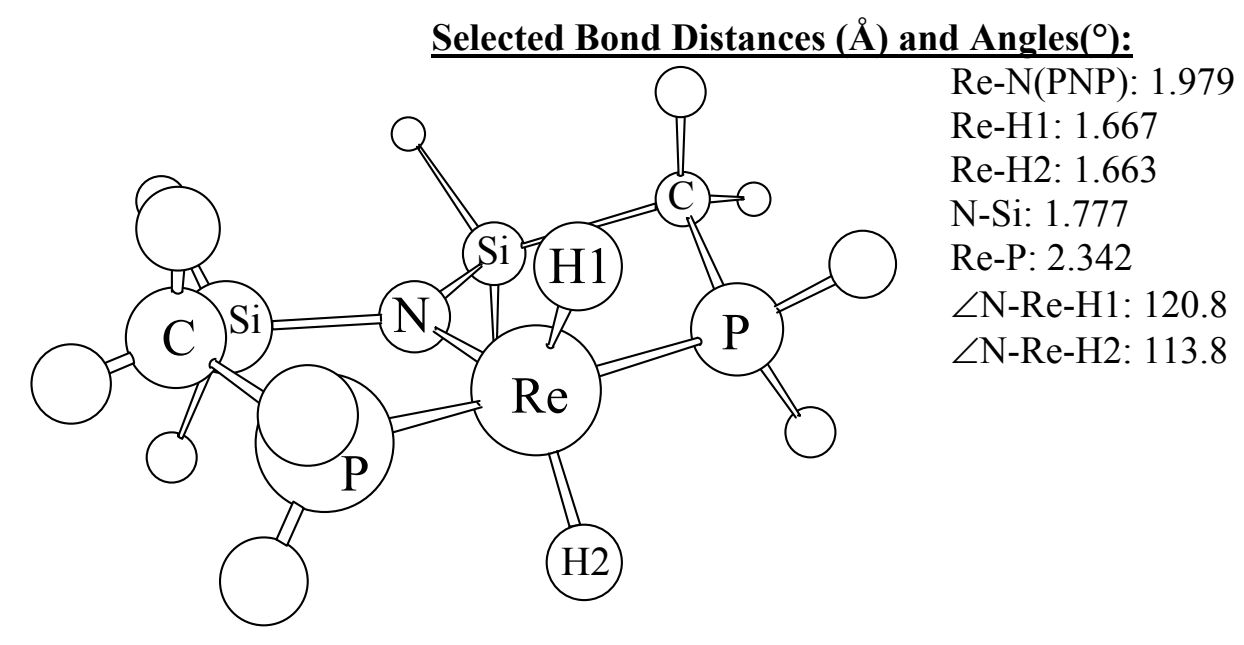

\section{Atomic Coordinates (XYZ)}

\begin{tabular}{llll}
\hline $\mathrm{C}-2.87611$ & 0.94490 & 0.52701
\end{tabular}

Si - $1.520702 .06457-0.14308$

N $0.00007 \quad 1.15052-0.04468$

Si $1.520802 .06473-0.14225$

$\begin{array}{llll}\text { C } 2.87631 & 0.94459 & 0.52683\end{array}$

P $2.34055-0.75901-0.01289$

Re $-0.00003-0.82815-0.03485$

P -2.34062 -0.75886-0.01245

$\mathrm{H}-1.838832 .44528-1.54550$

H -1.40256 $3.32199 \quad 0.64025$

H $1.40263 \quad 3.321370 .64233$

H $1.838832 .44686-1.54431$

$\mathrm{H}-3.87980 \quad 1.21027 \quad 0.18597$

H -2.85039 $0.97293 \quad 1.62111$

H $2.85107 \quad 0.97226 \quad 1.62094$

H 3.879891 .210000 .18548

$\mathrm{H}-3.10727-0.89877-1.21357$

H -3.15537 -1.62230 0.78702

H $3.15542-1.622650 .78623$

H $3.10690-0.89876-1.21420$

H - $0.00014-1.68742-1.46277$

H $0.00005-1.492451 .48929$ 
Pnprehcme3

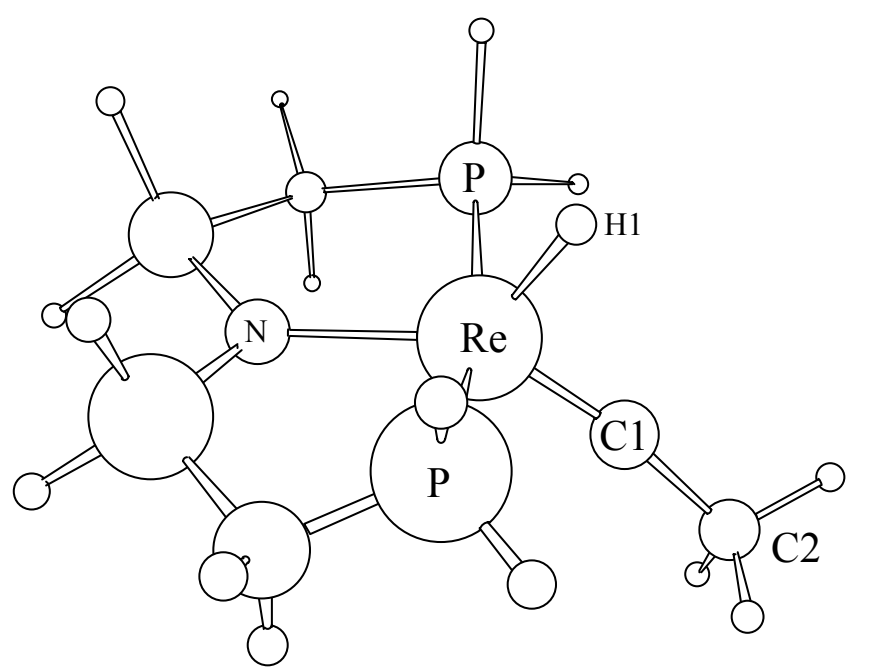

Selected Bond Distances $(\AA)$ and Angles $\left({ }^{\circ}\right):$

Re-N(PNP): 2.123

Re-H1: 1.649

Re-C1: 1.756

C1-C2: 1.469

N-Si: 1.750

Re-P: 2.378

$\angle \mathrm{N}-\mathrm{Re}-\mathrm{H} 1: 125.3$

$\angle \mathrm{Re}-\mathrm{C} 1-\mathrm{C} 2: 170.6$

$\angle \mathrm{N}-\mathrm{Re}-\mathrm{C} 1: 141.1$

\footnotetext{
Atomic Coordinates (XYZ)

Re $0.03451-0.62648-0.27358$

P -2.33958 $-0.52765-0.37248$

C -2.95990 $0.96706 \quad 0.53222$

$\begin{array}{llll}\mathrm{S} i-1.61821 & 2.28236 & 0.28356\end{array}$

H - $1.96653 \quad 3.07780-0.92872$

H - $1.63922 \quad 3.227801 .43432$

$\mathrm{N}-0.08374 \quad 1.453300 .13731$

$\begin{array}{lllll}\text { Si } & 1.35091 & 2.44838 & 0.26159\end{array}$

H $1.27153 \quad 3.40973 \quad 1.39667$

H $1.60433 \quad 3.25705-0.96526$

P $2.38257-0.27203-0.36068$

C $2.83125 \quad 1.29441 \quad 0.52424$

H -3.95629 $1.28645 \quad 0.21490$

H -2.99317 $0.71013 \quad 1.59648$

H 2.893361 .056691 .59168

H 3.785701 .719070 .20143

H -2.97679 -0.36901 -1.64113

H -3.14127 - 1.605020 .09915

H $3.28561-1.252160 .14054$

H $3.01676-0.07129-1.62494$

C $0.12097-2.178210 .54441$

H $0.07181-1.30089-1.77798$

C $0.19773-3.347181 .43139$

H $1.24872-3.574971 .65103$

H $-0.23684-4.233370 .95395$

H $-0.31271-3.176722 .38680$
} 


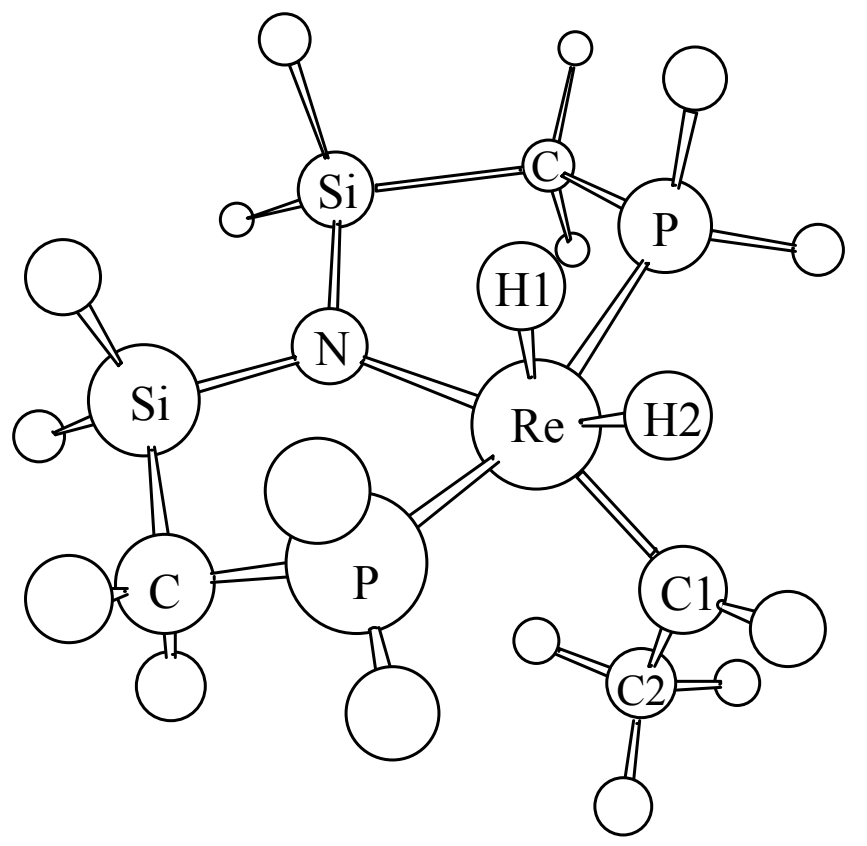

Selected Bond Distances $(\AA)$ and Angles $\left({ }^{\circ}\right):$

Re-N(PNP): 2.148

Re-H1: 1.633

Re-H2: 1.652

$\mathrm{H} 1-\mathrm{H} 2: 1.663$

Re-C1: 1.903

C1-C2: 1.514

N-Si: 1.745

Re-P: 2.380

$\angle \mathrm{N}-\mathrm{Re}-\mathrm{H} 1: 93.0$

$\angle \mathrm{N}-\mathrm{Re}-\mathrm{H} 2: 153.8$

$\angle \mathrm{Re}-\mathrm{C} 1-\mathrm{C} 2: 110.7$

$\angle \mathrm{N}-\mathrm{Re}-\mathrm{C} 1: 134.5$

\begin{abstract}
Atomic Coordinates (XYZ)
Re -0.00008 -0.69184 -0.28074

P - $2.35564-0.42330-0.48805$

C $-2.92480 \quad 1.10817 \quad 0.38716$

Si $-1.47581 \quad 2.32282 \quad 0.24522$

H - $1.679823 .12826-0.99417$

H - $1.530063 .27942 \quad 1.38683$

$\begin{array}{llll}\mathrm{N} & 0.00009 & 1.39201 & 0.23835\end{array}$

Si $1.476092 .32265 \quad 0.24628$

H 1.530443 .277861 .38905

H $1.680103 .12963-0.99213$

P $2.35537-0.42329-0.48882$

C $2.92503 \quad 1.107780 .38675$

$\mathrm{H}-3.87014 \quad 1.50076 \quad 0.00314$

H -3.05658 $0.84607 \quad 1.44259$

H $3.05751 \quad 0.84522 \quad 1.44198$

H 3.870151 .500510 .00232

H - $2.88905-0.23534-1.79587$

H -3.26344 -1.43484 -0.06121

H $3.26330-1.43504-0.06274$

H $2.88826-0.23487-1.79678$

C $0.00006-2.313700 .71561$

H $-0.00001-3.37785 \quad 0.47840$

C $0.00040-2.02810 \quad 2.20204$

H $0.88396-2.471522 .68148$

H - $0.88292-2.471582 .68187$

H $0.00040-0.952372 .43536$

H - $0.00022-1.95360-1.34642$
\end{abstract}

H $-0.00038-0.37951-1.88406$ 


\section{Pnprehcme-h2}

Selected Bond Distances $(\AA \AA)$ and

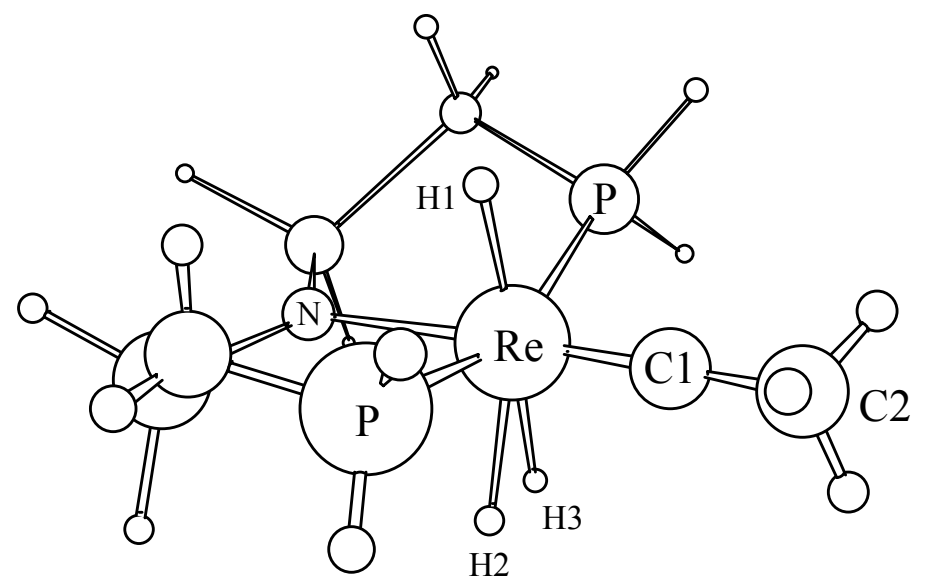
Angles( $\left.{ }^{\circ}\right)$ :

Re-N(PNP): 2.274

Re-H1: 1.681

Re-H2: 1.822

Re-H3: 1.822

H2-H3: 0.880

Re-C1: 1.763

C1-C2: 2.117

N-Si: 1.711

Re-P: 2.397

$\angle \mathrm{N}-\mathrm{Re}-\mathrm{H} 1: 86.4$

$\angle \mathrm{Re}-\mathrm{C} 1-\mathrm{C} 2: 179.1$

$\angle \mathrm{N}-\mathrm{Re}-\mathrm{C} 1: 177.9$

\footnotetext{
Atomic Coordinates (XYZ)

Re $0.64130-0.00027-0.10009$

$\begin{array}{lll}\text { P } 0.37176 & -2.35293 & 0.27047\end{array}$

C $-1.36883-2.719260 .75014$

Si $-2.42890-1.51018-0.27044$

H - $2.52344-2.10177-1.64263$

H -3.81394 - 1.505450 .28256

$\mathrm{N}-1.631920 .00060-0.17468$

Si $-2.42735 \quad 1.51212-0.27154$

H -3.81289 $1.50886 \quad 0.28021$

H -2.52001 2.10343 -1.64397

$\begin{array}{lllll}\text { P } & 0.37355 & 2.35234 & 0.27137\end{array}$

C $-1.36702 \quad 2.72023 \quad 0.74988$

$\mathrm{H}-1.61500-3.77701 \quad 0.62783$

$\mathrm{H}-1.47374-2.44201 \quad 1.80432$

H -1.47314 2.442991 .80394

H -1.61203 $3.77823 \quad 0.62747$

H $0.61661-3.30735-0.75949$

H $1.18416-2.951951 .27448$

H 1.185832 .950241 .27614

H $0.620023 .30688-0.75809$

H $0.48034-0.000581 .57276$

C $2.40448-0.00078-0.10619$

C $3.87094-0.00090-0.13454$

H $4.26449-0.884850 .38219$

$\begin{array}{llll}\text { H } & 4.26445 & 0.88460 & 0.37959\end{array}$

H $4.25615-0.00235-1.16100$

H $0.43415-0.43990-1.85627$

H $0.434380 .44013-1.85605$
} 


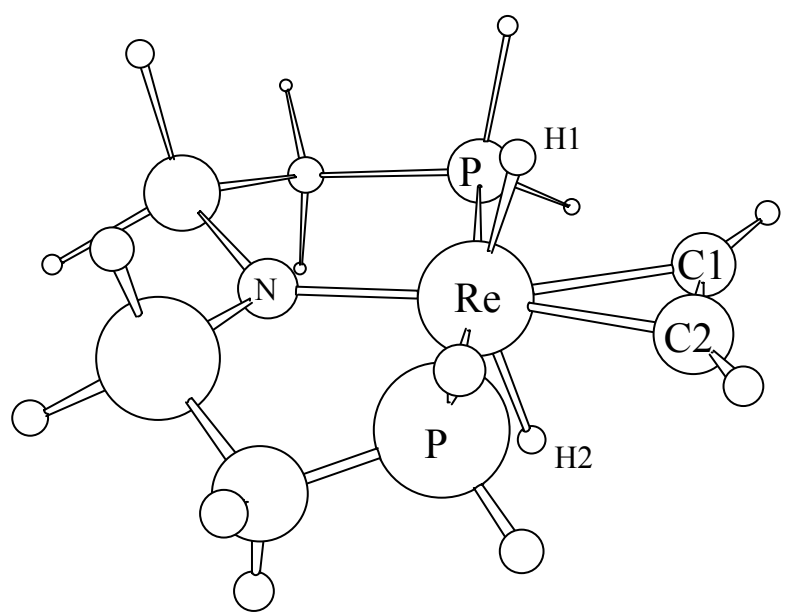

Atomic Coordinates (XYZ)

C $2.88775 \quad 1.24275-0.47344$

Si 1.489482 .327890 .16228

N -0.00206 1.374290 .03685

Si - $1.495342 .32602 \quad 0.15482$

C - $-2.89237 \quad 1.23412-0.47207$

P - 2.35401 -0.48471 -0.02185

Re $0.00085-0.629350 .01774$

P $2.35516-0.47848-0.02577$

H $1.749702 .72823 \quad 1.56961$

H $1.375693 .57320-0.64042$

H - $1.383983 .56480-0.65818$

H - $1.755632 .73773 \quad 1.55894$

H $3.873521 .50596-0.08276$

H $2.909441 .30872-1.56602$

H -2.91863 $1.29686-1.56474$

H $-3.87738 \quad 1.49587-0.07847$

H $3.12269-0.712291 .15711$

H $3.13044-1.30272-0.89555$

H -3.12874 -1.31249 -0.88873

H -3.11887 -0.71848 1.16277

H $0.00248-1.052321 .64343$

H $0.00070-1.14690-1.58992$

C $0.63381-2.77490 \quad 0.08647$

C $-0.62528-2.77685 \quad 0.08654$

H $1.56222-3.318520 .09746$

H - 1.55181 -3.32365 0.09733
Selected Bond Distances $(\AA)$ and Angles $\left({ }^{\circ}\right):$

Re-N(PNP): 2.004

Re-H1: 1.680

Re-H2: 1.689

Re-C1: 2.238

$\mathrm{Re}-\mathrm{C} 2: 2.238$

C1-C2: 1.259

N-Si: 1.775

Re-P: 2.360

$\angle \mathrm{N}-\mathrm{Re}-\mathrm{H} 1: 104.0$

$\angle \mathrm{N}-\mathrm{Re}-\mathrm{H} 2: 108.4$

$\angle \mathrm{Re}-\mathrm{C} 1-\mathrm{C} 2: 73.7$

$\angle \mathrm{N}-\mathrm{Re}-\mathrm{C} 1: 163.5$ 


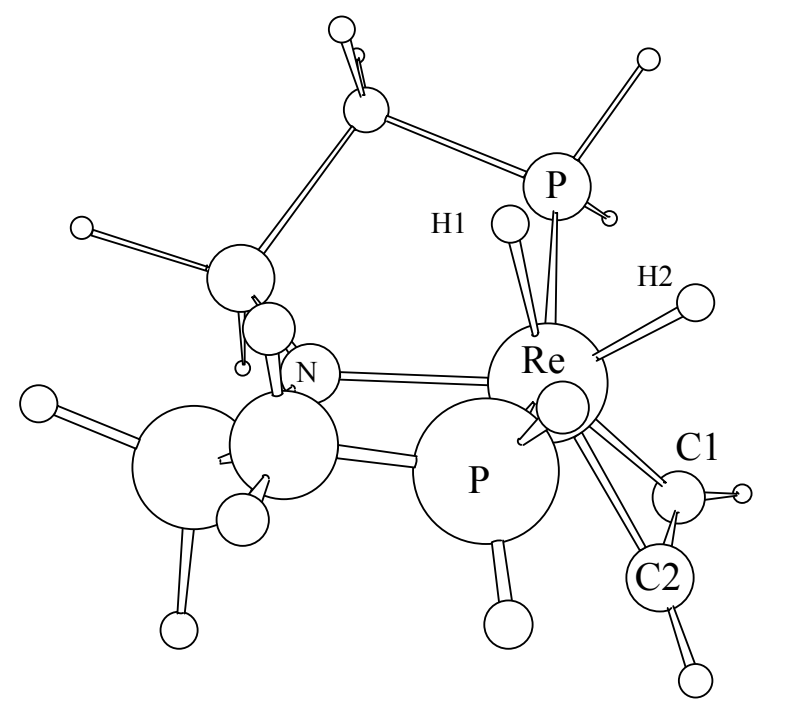

Selected Bond Distances $(\AA)$ and Angles $\left({ }^{\circ}\right):$ Re-N(PNP): 2.131

Re-H1: 1.702

Re-H2: 1.657

$\mathrm{Re}-\mathrm{C} 1: 2.013$

Re-C2: 2.013

C1-C2: 1.315

N-Si: 1.733

Re-P: 2.379

$\angle \mathrm{N}-\mathrm{Re}-\mathrm{H} 1: 81.3$

$\angle \mathrm{N}-\mathrm{Re}-\mathrm{H} 2: 146.4$

$\angle \mathrm{Re}-\mathrm{C} 1-\mathrm{C} 2: 70.9$

$\angle \mathrm{N}-\mathrm{Re}-\mathrm{C} 1: 120.7$

\begin{abstract}
Atomic Coordinates (XYZ)
C -2.70179 $1.36422-0.71578$

Si - $1.514662 .17288 \quad 0.52227$

N -0.00018 1.349350 .34232

Si $1.514162 .17303 \quad 0.52271$

C $2.70161 \quad 1.36493-0.71539$

P $2.29973-0.43419-0.58263$

Re $0.00005-0.74340-0.05688$

P -2.29988 -0.43480 -0.58198

C $-0.65658-2.05425 \quad 1.32263$

C $0.65800-2.054041 .32219$

$\mathrm{H}-1.50916-2.56327 \quad 1.74950$

H $1.51112-2.56277 \quad 1.74825$

H $0.00011-1.92710-1.21613$

H - $0.00048-0.17445-1.66105$

H -3.29063 -0.908090 .32481$

H -2.82693 -1.02105 -1.76839

H -3.76133 $1.57435-0.55212$

H -2.42103 $1.69121-1.72240$

H - $1.41411 \quad 3.63253 \quad 0.24409$

H -2.12256 2.031261 .87761

H $2.421171 .69244-1.72193$

H 1.413393 .632750 .24495

H $3.761111 .57498-0.55134$

H $2.121882 .03111 \quad 1.87810$

H $3.29082-0.908270 .32335$

H 2.82632 -1.01955-1.76969
\end{abstract}




\section{Pnpreh3ph}

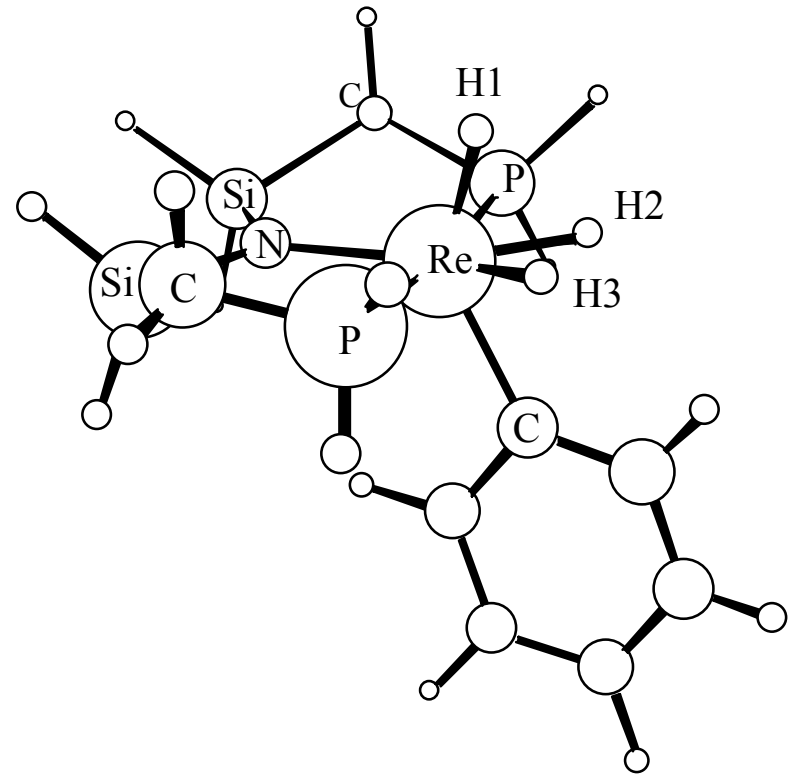

Selected Bond Distances $(\AA)$ and Angles( $\left.{ }^{\circ}\right)$ :

Re-N(PNP): 2.069

Re-H1: 1.643

Re-H2: 1.660

Re-H3: 1.660

H2-H3: 1.455

Re-C: 2.102

Re-P: 2.382

$\angle \mathrm{N}-\mathrm{Re}-\mathrm{H} 1: 107.6$

$\angle \mathrm{N}-\mathrm{Re}-\mathrm{C}: 116.9$

\section{Atomic Coordinates (XYZ)}

C - $1.86415 \quad 2.87905 \quad 0.11540$

Si $-2.27018 \quad 1.48580 \quad 1.32027$

N - 1.634710 .000090 .62526

Si -2.27074 -1.48539 1.32023

C - $1.86444-2.87893 \quad 0.11579$

P $-0.25597-2.37374-0.64836$

Re $-0.08182-0.00007-0.74263$

P $-0.255832 .37363-0.64890$

$\mathrm{H}-1.65051 \quad 1.788822 .63986$

H -3.73907 1.400701 .53329

H -3.73971 $-1.39997 \quad 1.53259$

H - $1.65173-1.788342 .64016$

H - $1.82677 \quad 3.87152 \quad 0.57124$

H -2.61869 $2.88114-0.67797$

H $-2.61880-2.88129-0.67775$

$\mathrm{H}-1.82712 \quad-3.87127 \quad 0.57190$

H $0.69605 \quad 3.102900 .12795$
H - $0.169153 .14582-1.84012$

H $-0.16915-3.14620-1.83939$

H $0.69576-3.102890 .12878$

C $1.87136-0.00003 \quad 0.03494$

H $0.88869-0.72760-1.87617$

H $-0.74401-0.00020-2.24660$

H $0.888720 .72713-1.87637$

C $3.12594-0.00041-0.59320$

C $4.31461-0.000380 .13776$

C $4.290540 .00001 \quad 1.53010$

C $3.05767 \quad 0.000392 .17999$

$\begin{array}{llll}\text { C } & 1.87079 & 0.00038 & 1.44719\end{array}$

H $3.17884-0.00072-1.67943$

H $5.26603-0.00067-0.38950$

H $5.21508 \quad 0.00003 \quad 2.10071$

H 3.012220 .000703 .26679

H 0.928790 .000691 .99453 


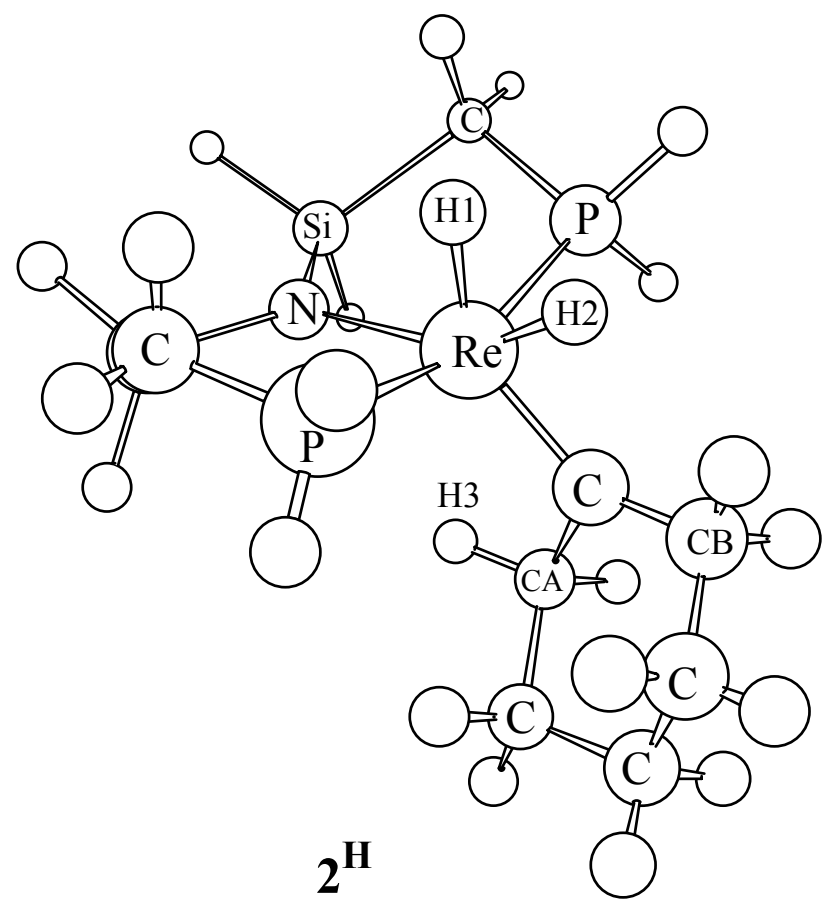

Selected Bond Distances $(\AA)$ and Angles( $\left(^{\circ}\right)$ :

Re-N(PNP): 2.183

Re-C: 1.897

Re-H1: 1.644

Re-H2: 1.649

Re-H3: 2.471

Re-CA: 2.672

N-Si: 1.728

Re-P: 2.375(av)

C-CA: 1.527

C-CB: 1.493

CA-H3: 1.108

$\angle \mathrm{N}-\mathrm{Re}-\mathrm{H} 1: 90.4$

$\angle \mathrm{N}-\mathrm{Re}-\mathrm{H} 2: 152.2$

$\angle \mathrm{N}-\mathrm{Re}-\mathrm{C}: 135.2$
Atomic Coordinates (XYZ)

C $1.77354-0.498031 .42261$

C $1.77375-0.58677-0.10221$

C $3.10315-0.93145-0.68691$

C $4.209450 .00624-0.16414$

$\begin{array}{llll}\text { C } 4.23663 & 0.05478 & 1.36431\end{array}$

C $2.87300 \quad 0.44877 \quad 1.93355$

Re -0.02676 -0.15997-0.52202

P $0.178752 .17781-0.89784$

C - $1.446672 .99561-0.57100$

Si -2.15922 $2.04128 \quad 0.90711$

$\mathrm{N}-1.801220 .37304 \quad 0.63312$

Si $-2.82263-0.88648 \quad 1.23026$

C -2.69014 -2.32215-0.00517

P $-0.89752-2.36665-0.45411$

H $-0.82226-3.22376-1.59268$

$\mathrm{H}-0.38278-3.295550 .50226$

$\mathrm{H}-3.24692-2.04597-0.90695$

$\mathrm{H}-3.05823-3.28346 \quad 0.36223$

H $-4.25173-0.48302 \quad 1.36233$
H -2.41167 -1.42285 2.56385

H -3.61814 2.32758 1.01700

H - 1.524642 .607422 .13666

H -2.08790 $2.80410-1.43795$

$\mathrm{H}-1.355454 .07485-0.42405$

H $0.592312 .67291-2.17080$

H $1.060802 .97417-0.10522$

H -0.89105 -0.06227 -1.91694

H $0.75148-0.46638-1.94289$

H $3.05524-0.91569-1.77835$

H $3.35350-1.96242-0.38443$

H $4.032061 .01575-0.55813$

H $5.17952-0.31821-0.55796$

H 5.005310 .757561 .70710

H $4.52128-0.932761 .75496$

H 2.629721 .474941 .62970

H 2.891050 .439673 .02965

H $1.93897-1.508701 .82833$

H $0.78628-0.17801 \quad 1.81150$ 


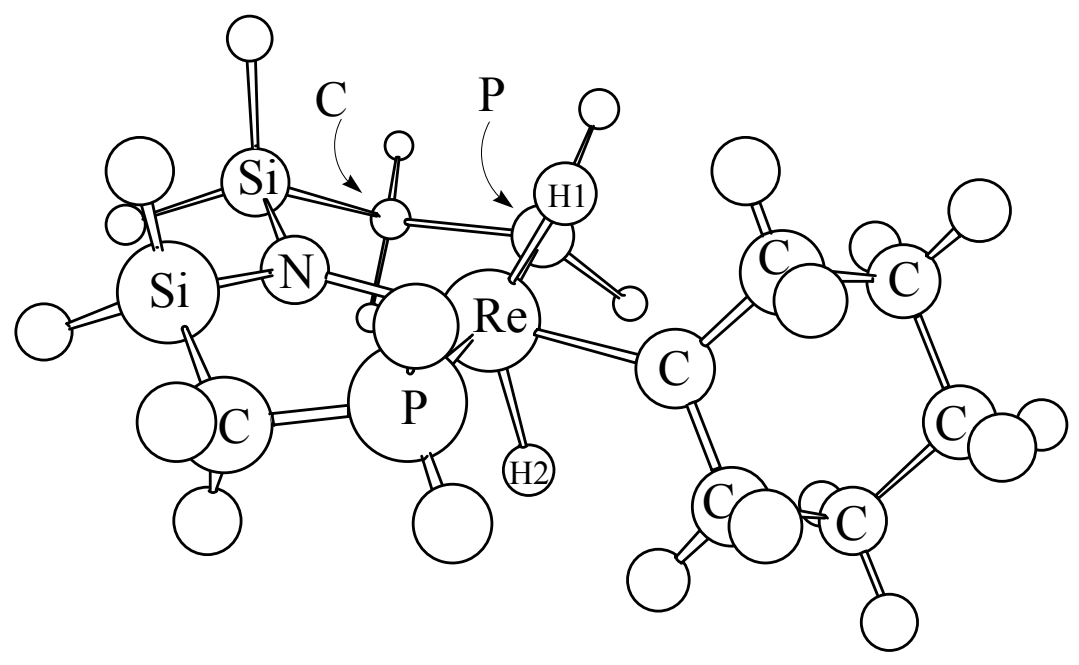

Selected Bond Distances

$(\AA)$ and Angles $\left({ }^{\circ}\right)$ :

Re-N(PNP): 2.079

Re-C: 2.026

Re-H1: 1.633

Re-H2: 1.644

N-Si: 1.758

Re-P: 2.370(av)

$\angle \mathrm{N}-\mathrm{Re}-\mathrm{H} 1: 108.7$

$\angle \mathrm{N}-\mathrm{Re}-\mathrm{H} 2: 113.6$

$\angle \mathrm{N}-\mathrm{Re}-\mathrm{C}: 174.5$

\section{$\mathbf{i}-2^{\mathrm{H}}$}

\section{Atomic Coordinates (XYZ)}

Re -0.08243 -0.06800 0.03596

$\mathrm{N}-2.14113 \quad 0.21788 \quad 0.02852$

P $-0.57154-2.384990 .07433$

P $0.106262 .29365-0.07560$

C - $2.33097-2.66817-0.44617$

C - $1.52463 \quad 3.01780-0.59257$

Si -3.26061 -1.13138 0.14057

Si $-2.82609 \quad 1.835550 .09751$

H $0.17002-3.32835-0.70210$

H $-0.52629-3.113981 .30394$

H -2.35182 -2.68551-1.54098

H -2.74229 -3.60899 -0.07166

H $-4.46707-0.91492-0.70393$

H -3.73915 -1.35573 1.53269

H $-4.075641 .93672-0.70504$

H -3.16597 2.243751 .48815

H - $1.568922 .97457-1.68601$

$\mathrm{H}-1.651274 .05645-0.27664$

H $1.044112 .93753-0.93793$
H $0.39501 \quad 3.065881 .09212$

H $0.45323 \quad 0.02404 \quad 1.57540$

H $0.57615-0.15646-1.46794$

C $1.89232-0.505160 .15161$

C $2.74774-1.05483-0.97741$

C $3.89464-0.08661-1.33204$

C $4.785940 .17581-0.12048$

$\begin{array}{llll}\text { C } 3.95192 & 0.61737 & 1.07941\end{array}$

C $2.79423-0.363701 .36492$

H $2.14842-1.26717-1.86561$

H $3.20534-2.00301-0.64675$

H $3.462090 .85744-1.68926$

H $4.47785-0.49987-2.16365$

H $5.545760 .93080-0.35745$

H $5.32789-0.745640 .13524$

H 3.529101 .613130 .88772

H $4.576300 .71077 \quad 1.97600$

H $3.23596-1.345851 .60462$

H $2.23712-0.033812 .24475$ 


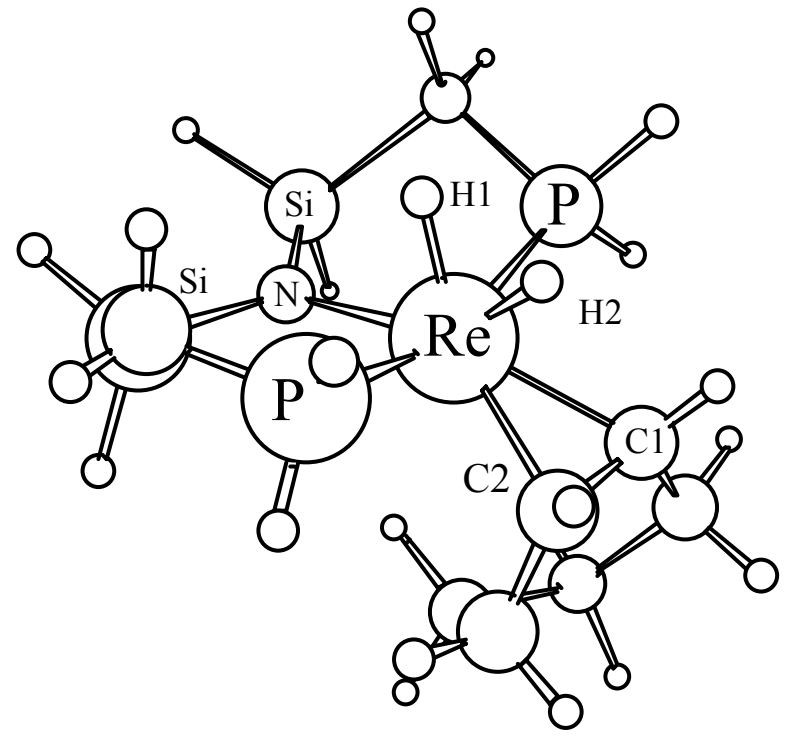

$4^{\mathrm{H}}$
Selected Bond Distances $(\AA)$ and Angles( $\left(^{\circ}\right):$

Re-N(PNP): 2.136

Re-C1: 2.249

$\mathrm{Re}-\mathrm{C} 2: 2.226$

C1-C2: 1.430

Re-H1: 1.630

Re-H2: 1.644

N-Si: 1.737

Re-P: 2.360(av)

$\angle \mathrm{N}-\mathrm{Re}-\mathrm{H} 1: 81.8$

$\angle \mathrm{N}-\mathrm{Re}-\mathrm{H} 2: 141.2$

$\angle \mathrm{N}-\mathrm{Re}-\mathrm{C} 1: 142.8$

$\angle \mathrm{N}-\mathrm{Re}-\mathrm{C} 2: 139.4$

$\underline{\text { Atomic Coordinates (XYZ) }}$ 
C $1.17312 \quad 3.08254-0.44226$

Si $1.853602 .07534 \quad 1.00810$

N $1.543920 .40928 \quad 0.62581$

Si $2.64531-0.83607 \quad 1.13019$

C $2.61642-2.15944-0.22229$

P $0.82759-2.24531-0.69259$

Re $-0.16210-0.10633-0.55098$

P - $0.422172 .22183-0.81836$

C -2.40694 $0.01342-0.60054$

C - $-2.00522-1.34529-0.40442$

H -2.76268 $0.28758-1.59085$

H -2.14167 -2.05607 -1.21615

H - $0.62317-0.32048-2.11474$

H $0.892900 .14663-1.76734$

H - $1.330242 .99455-0.02845$

$\mathrm{H}-0.828142 .73740-2.08714$

H $1.046674 .15034-0.24729$

H $1.839272 .94741-1.30058$

H 3.301292 .363741 .20766

H 1.151892 .544072 .24019

H $3.17243-1.78078-1.08628$

H $4.03475-0.336701 .32780$

H $3.02671-3.128940 .07062$

H $2.24397-1.493922 .40964$

H $0.38722-3.35690 \quad 0.09390$

H $0.81771-2.91970-1.95093$

C $-3.16234 \quad 0.71521 \quad 0.52011$

C - $2.64641 \quad 0.42576 \quad 1.94540$

C - $1.72852-0.801002 .00231$

C $-2.14656-1.88594 \quad 1.00743$

H -3.20906 1.796090 .34514

H -4.20349 $0.36814 \quad 0.44057$

H -2.10783 1.292092 .34477

H -3.50449 0.269652 .60991

H $-0.68504-0.497201 .76922$

H - $1.67190-1.193823 .02373$

H - $1.53471-2.78411 \quad 1.14722$

H -3.18927 -2.18836 1.19599 


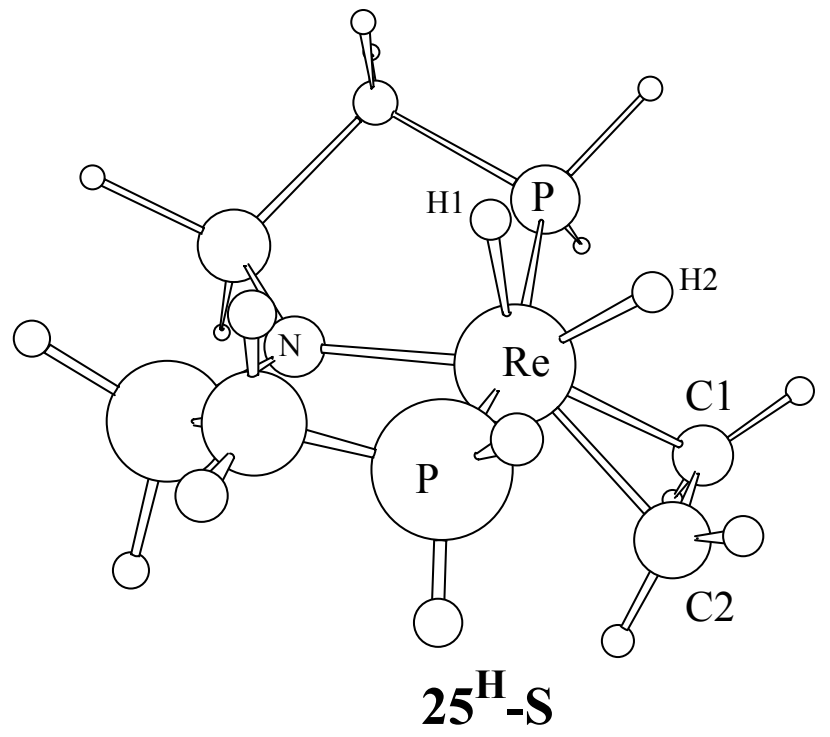

Selected Bond Distances $(\AA)$ and Angles $\left({ }^{\circ}\right):$ Re-N(PNP): 2.110

Re-H1: 1.630

Re-H2: 1.651

Re-C1: 2.203

Re-C2: 2.203

C1-C2: 1.426

N-Si: 1.746

Re-P: 2.360

$\angle \mathrm{N}-\mathrm{Re}-\mathrm{H} 1: 84.3$

$\angle \mathrm{N}-\mathrm{Re}-\mathrm{H} 2: 144.6$

$\angle \mathrm{Re}-\mathrm{C} 1-\mathrm{C} 2: 71.1$

$\angle \mathrm{N}-\mathrm{Re}-\mathrm{C} 1: 136.7$

Atomic Coordinates (XYZ)

Re $0.00008-0.66207-0.08742$ 
C $-0.71285-2.49863 \quad 0.89905$

C $0.71359-2.498400 .89903$

H - $1.25753-3.20550 \quad 0.28070$

H $1.25854-3.205120 .28076$

H - $1.22331-2.234521 .82652$

H $1.22398-2.234041 .82648$

H $0.00017-1.78896-1.29339$

H $-0.00007-0.17932-1.64463$

$\begin{array}{llll}\mathrm{N}-0.00019 & 1.40541 & 0.33275\end{array}$

P -2.33030 -0.41087 -0.36092

C -2.74685 $1.36905-0.64649$

Si - $1.506612 .28001 \quad 0.45401$

$\begin{array}{lllll}\text { Si } & 1.50620 & 2.27999 & 0.45459\end{array}$

C $2.746351 .36980-0.64664$

P $2.33030-0.41026-0.36129$

H -3.23842 -0.743120 .69300$

H -3.00807 -1.11964-1.39870

H -3.79557 $1.60282-0.44736$

$\mathrm{H}-2.517551 .60371-1.69118$

H - $1.38415 \quad 3.69906 \quad 0.01974$

H -2.05293 2.30132 1.84147

H $2.516461 .60464-1.69117$

H $1.383703 .69938 \quad 0.02140$

H $3.795081 .60385-0.44792$

H 2.052592 .300241 .84203

H $3.23871-0.742510 .69239$

H $3.00797-1.11873-1.39935$ 


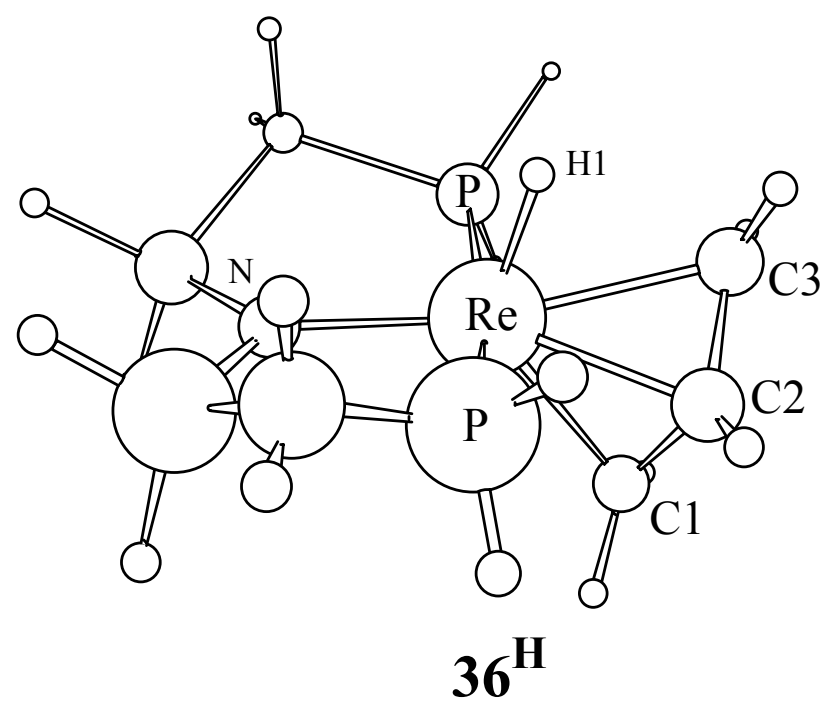

Selected Bond Distances $(\AA)$ and

Angles $\left({ }^{\circ}\right)$ :

Re-N(PNP): 2.030

Re-H1: 1.648

Re-C1: 2.178

Re-C2: 2.244

Re-C3: 2.378

C1-C2: 1.437

C2-C3: 1.402

N-Si: 1.765

Re-P: 2.350

$\angle \mathrm{N}-\mathrm{Re}-\mathrm{H} 1: 109.5$

$\angle \mathrm{Re}-\mathrm{C} 1-\mathrm{C} 2: 68.6$

$\angle \mathrm{N}-\mathrm{Re}-\mathrm{C} 1: 124.3$

$\angle \mathrm{N}-\mathrm{Re}-\mathrm{C} 2: 156.9$

$\angle \mathrm{Re}-\mathrm{C} 2-\mathrm{C} 3: 77.7$

\footnotetext{
Atomic Coordinates (XYZ)

C - $1.25952-2.55631-0.57616$

C $-0.33870-2.674390 .47423$

C $-0.58440-1.82190 \quad 1.60481$

$\mathrm{H}-1.05878-3.04480-1.52431$

$\mathrm{H}-2.31455-2.41694-0.35163$

H $0.55572-3.286610 .38445$

H $0.16757-1.816512 .39452$

H -1.60641 -1.73949 1.97846

Re $-0.16044-0.50738-0.07861$

P $2.11786-1.07031-0.18324$

C $3.133030 .43903-0.55461$

Si $2.15796 \quad 1.83927 \quad 0.24559$

N $0.45067 \quad 1.420590 .09956$

$\begin{array}{llll}\text { Si }-0.70017 & 2.74353 & 0.29911\end{array}$

C - $2.32432 \quad 2.17979-0.47135$

P -2.34764 $0.34630-0.17124$

H $2.80422-1.589260 .96641$

H $2.62257-2.04161-1.10557$

H $4.167960 .35692-0.21401$

H $3.127130 .58510-1.63976$

H $2.576291 .97961 \quad 1.66768$

H $2.456303 .13411-0.42391$

H - $0.209443 .98397-0.35917$

H - 0.923503 .072991 .73383

$\mathrm{H}-2.270042 .33901-1.55311$

H -3.20919 2.68946-0.08279

H -3.23164 0.26832 0.95931

H -3.29315 -0.13590 -1.12946

H $-0.20165-0.92548-1.67200$
} 


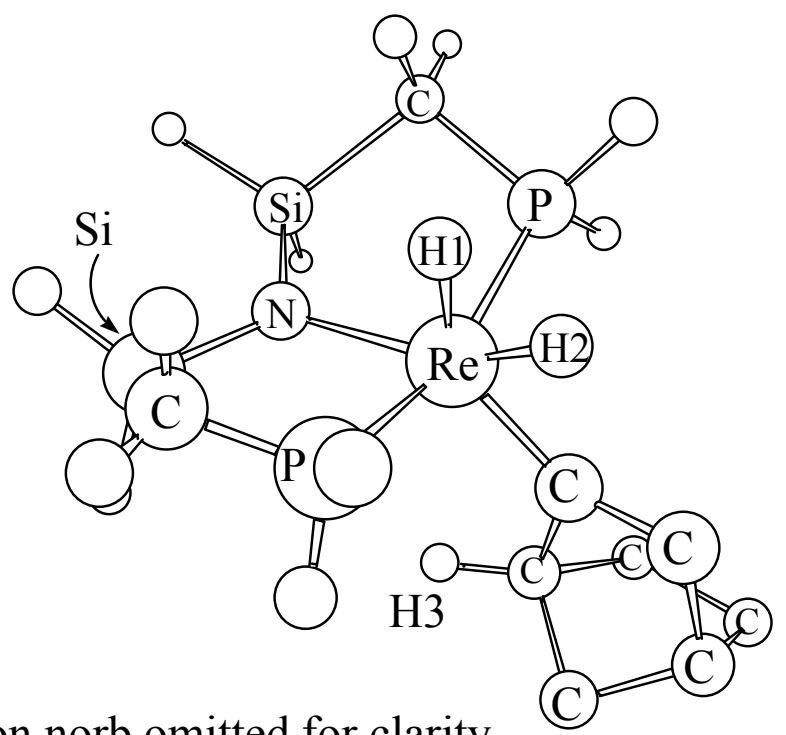

Selected Bond Distances $(\AA)$ and

Angles $\left({ }^{\circ}\right)$ :

Re-N(PNP): 2.158

Re-H1: 1.643

Re-H2: 1.657

Re-C1: 1.904

Re-H3: 2.848

C-H3: 1.096

N-Si: 1.735

Re-P: 2.370

$\angle \mathrm{N}-\mathrm{Re}-\mathrm{H} 1: 46.1$

$\angle \mathrm{N}-\mathrm{Re}-\mathrm{H} 2: 136.1$

$\angle \mathrm{N}-\mathrm{Re}-\mathrm{C} 1: 133.9$

H's on norb omitted for clarity

\section{Atomic Coordinates (XYZ)}

C - $1.71832-0.17116-0.28396$

Re $0.15884-0.04802-0.58056$

C -3.04872 -0.40361 -0.96697

$\begin{array}{llll}\text { N } & 1.87204 & 0.10529 & 0.72257\end{array}$

P $0.37098 \quad 2.30348-0.82551$

C $2.067232 .82613-0.30123$

Si $2.484351 .67214 \quad 1.14543$

Si $2.64985-1.33763 \quad 1.28852$

C $2.35262-2.66621-0.03290$

P $0.61138-2.37429-0.58560$

C -2.06837 $-0.14064 \quad 1.21277$

C - $4.05551-0.528440 .19345$

C $-3.18198-1.21174 \quad 1.26208$

C - $2.88036 \quad 1.15691 \quad 1.47237$

C $-4.254570 .87068 \quad 0.80445$

H - $0.59311-0.16691-2.05218$

H $1.07742-0.07000-1.94255$

H -0.44374 $3.19265-0.05930$

H $0.170852 .93386-2.09067$

H $2.129233 .89290-0.07134$

H $2.751562 .59828-1.12538$

H 1.858852 .251652 .37172

H 3.957051 .687811 .37580

H $4.11645-1.175351 .49920$

H $2.09299-1.855742 .57408$

H $2.52770-3.692950 .29835$

H $3.00699-2.44816-0.88368$

H $0.47161-3.14803-1.77735$

H - $0.11047-3.254230 .27862$

H - $2.98426-1.32611-1.55773$

H -3.28007 $0.40021-1.67601$
H $-1.22452-0.27327 \quad 1.89901$

H -3.66216 -1.27598 2.24532

H $-4.98557-1.03975-0.06899$

H -5.05929 $0.86034 \quad 1.54719$

$\begin{array}{llll}\mathrm{H}-4.52052 & 1.61557 & 0.04745\end{array}$

H -2.98433 $1.31452 \quad 2.55140$

H -2.38152 2.039101 .06253

H -2.83534 -2.20838 0.96934 


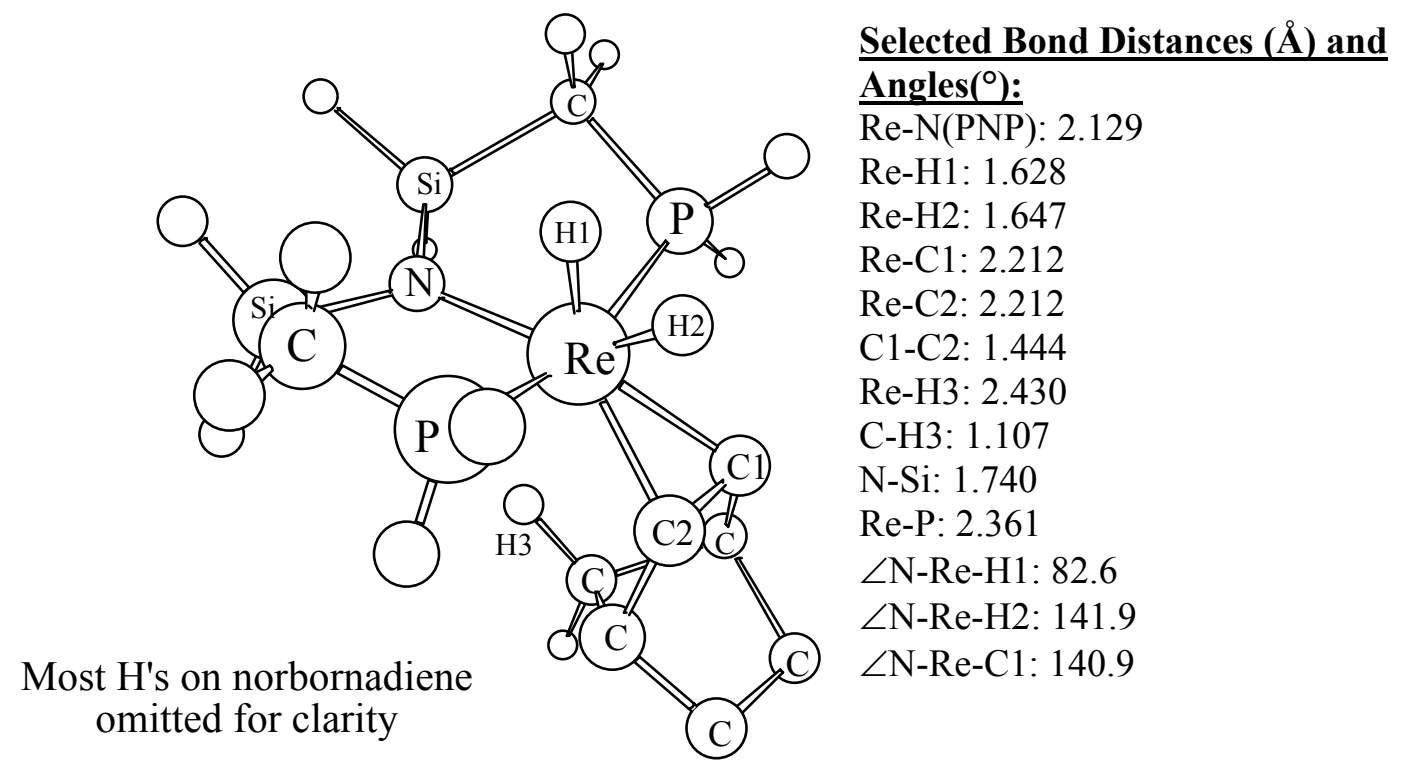

\section{Atomic Coordinates (XYZ)}

C - $2.036810 .72151-0.60383$

C - $2.03664-0.72201-0.60356$

C $-2.55255 \quad 1.128620 .77664$

C $-2.55233-1.128650 .77709$

C $-1.97206 \quad 0.00021 \quad 1.64513$

C $-4.06213 \quad 0.778680 .80446$

C $-4.06197-0.77900 \quad 0.80475$

$\mathrm{H}-2.354841 .29310-1.47177$

H - $2.35447-1.29393-1.47134$

H -2.32151 2.14986 1.09133

H - $2.32114-2.149721 .09225$

$\mathrm{H}-0.86605 \quad 0.00031 \quad 1.69390$

H -2.34085 0.000392 .67652

$\mathrm{H}-4.59308 \quad 1.20265-0.05379$

$\mathrm{H}-4.535371 .176481 .70946$

$\mathrm{H}-4.59284-1.20340-0.05333$

H -4.53512 -1.17655 1.70991

Re $0.05386-0.00007-0.55535$

$\mathrm{N} 1.77387 \quad 0.00013 \quad 0.69944$

H $1.174570 .00001-1.73642$

H -0.39294 -0.00032 -2.14020

P $0.40197 \quad 2.33075-0.69102$

C $2.137592 .74508-0.20199$

Si 2.496301 .506901 .18380

Si $2.49683-1.50641 \quad 1.18374$

C $2.13807-2.74471-0.20194$

P $0.40231-2.33084-0.69073$

H $0.198873 .00049-1.93577$

H - $0.337713 .24845 \quad 0.11837$
H $2.789052 .51028-1.05027$

H 2.268183 .794690 .07272

H 3.965101 .398341 .40679

H 1.915682 .061742 .44243

H $3.96567-1.397531 .40625$

H $1.91670-2.061292 .44257$

H $2.78929-2.50965-1.05034$

H $2.26909-3.794290 .07269$

H $0.19919-3.00073-1.93541$

H $-0.33710-3.248660 .11878$ 


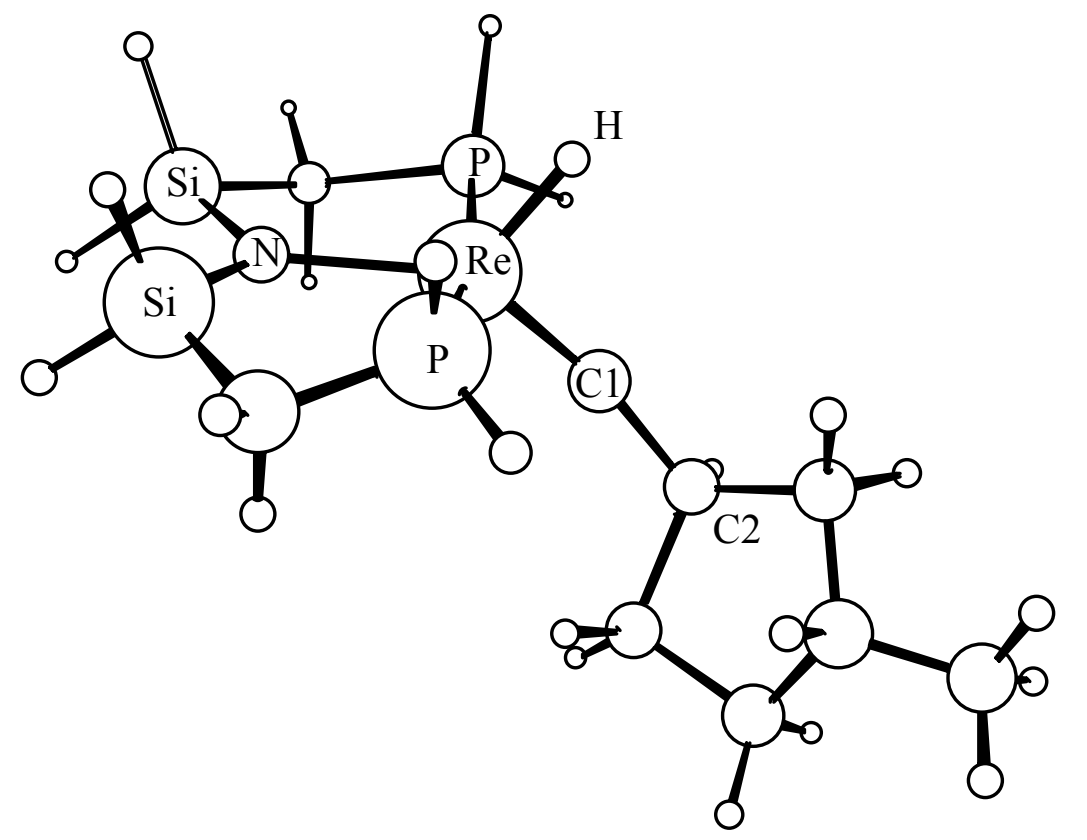

Selected Bond Distances

$(\AA)$ and Angles $\left({ }^{\circ}\right)$ :

Re-N(PNP): 2.124

Re-H1: 1.650

Re-C1: 1.759

C1-C2: 1.470

N-Si: 1.750

Re-P: 2.376

$\angle \mathrm{N}-\mathrm{Re}-\mathrm{H} 1: 125.6$

$\angle \mathrm{N}-\mathrm{Re}-\mathrm{C} 1: 140.8$

$\angle \mathrm{Re}-\mathrm{C} 1-\mathrm{C} 2: 170.3$

\section{Atomic Coordinates (XYZ)}

C $3.73846-0.64069-0.56543$

C $4.70804 \quad 0.292920 .18648$

H $4.88235-0.956851 .94787$

C $2.89366-0.123631 .70684$

C $2.53782-0.896080 .40500$

C $1.22098-0.56329-0.15644$

H $2.50021-1.971040 .63753$

H $3.40172-0.21861-1.51568$

H $4.23413-1.59158-0.79392$

C $6.167140 .13597-0.22343$

H $4.400601 .33137-0.00696$

H 4.806970 .765532 .34016

H $2.50857-0.625532 .59910$

H 2.437920 .872601 .67249

H $6.51714-0.88567-0.03281$

H $6.81530 \quad 0.820180 .33487$

H $6.307950 .34163-1.28990$

Re -0.44358 -0.14941 -0.54600

$\begin{array}{lllll}\mathrm{N}-2.27154 & 0.38028 & 0.39673\end{array}$

P - $1.25205-2.37790-0.40639$

C - $2.56351-2.504050 .89830$

$\begin{array}{llll}\text { Si }-3.44659 & -0.82779 & 0.86774\end{array}$

Si -2.72154 2.050490 .66094

C - $1.15243 \quad 3.09968 \quad 0.49232$

P -0.10739 $2.19653-0.74398$

H $1.187972 .77434-0.62588$

$\mathrm{H}-0.485162 .85157-1.95630$

$\mathrm{H}-0.618803 .06907 \quad 1.44844$

H - $1.34323 \quad 4.14276 \quad 0.22564$

H -3.71983 $2.54723-0.32937$
H -3.315042 .268062 .00972$

H -4.59417 -0.92385 -0.07986

H -4.02696 -0.554442 .21214$

H -3.23111 -3.35972 0.76565

H -1.91744 -2.95925 -1.52901

H -2.05107 -2.60020 1.86164

H $-0.36419-3.45984-0.14332$

H $-0.21624-0.32041-2.17102$

C $4.41606-0.002661 .6640$ 


\section{File Name}

Energy (a.u.)
E with ZPE

ZPE (kcal/mol) (kcal/mol)
$\mathrm{T}(\mathrm{S})$

(cal/molK) $\mathrm{kcal} / \mathrm{mol}$
G at $298.15 K$

(a.u.) pnpreh2-s (3H)

pnpreh4 $(1 \mathrm{H})$

pnpreh2cy-s (i-2H)

pnpreh2cy $(2 \mathrm{H})$

pnpcyclohexene

$(4 \mathrm{H})$

h2

cy-carb

\begin{abstract}
$-239.4336259$
$-240.6537784$

$-474.0210785$

$-474.0489134$

$-474.0501637$

$-1.177516506$

$-234.4557785$
\end{abstract}

94.23135

105.51360

188.30098

188.28830

188.98823

6.36934

90.67465

\begin{abstract}
$-150,152.65$
$-150,907.03$

$-297,264.45$

$-297,281.93$

$-297,282.01$

$-732.53$

$-147,032.56$
\end{abstract}

112.047

114.137

147.389

143.780

140.149

31.136

74.256
33.41

34.03

43.94

42.87

41.79

9.28

22.14
$-239.323094$

$-240.525771$

$-473.770874$

$-473.797098$

$-473.796000$

$-1.178855$ $-234.339931$ 
i Gaussian 98 (Revision A.7), M. J. Frisch, G. W. Trucks, H. B. Schlegel, G. E. Scuseria, M. A. Robb, J. R. Cheeseman, V. G. Zakrzewski, J. A. Montgomery, R. E. Stratmann, J. C. Burant, S. Dapprich, J. M. Milliam, A. D. Daniels, K. N. Kudin, M. C. Strain, O. Farkas, J. Tomasi, V. Barone, M. Cossi, R. Cammi, B. Mennucci, C. Pomelli, C. Adamo, S. Clifford, J. Ochterski, G. A. Petersson, P. Y. Ayala, Q. Cui., K. Morokuma, D. K. Malick, A. D. Rabuck, K. Raghavavhari, J. B. Foresman, J. Cioslowski, J. V. Ortiz, B. B. Stefanov, G. Liu, A. Liashenko, P. Piskorz, I. Komaromi, R. Gomperts, R. L. Martin, D. J. Fox, T. Keith, M. A. AlLaham, C. Y. Peng, A. Nanayakkara, C. Gonzalez, M. Challacombe, P. M. W. Gill, B. G. Johnson, W. Chen, M. W. Wong, J. L. Andres, M. Head-Gordon, E. S. Replogle and J. A. Pople, Gaussian, Inc., Pittsburgh PA, 1998.

ii $\quad$ Becke, A. D. Phys. Rev. 1988, A38, 3098.; Becke, A. D. J. Chem. Phys. 1993, 98, 1372.; Becke, A. D. J. Chem. Phys. 1993, 98, 5648.; Perdue, J. P.; Wang, Y. Phys. Rev. B. 1992, 45, 13244.

iii $\quad$ Hariharan, P. C.; Pople, J. A. Theor. Chim. Acta 1973, 28, 213.

iv $\quad$ Hay, P. J.; Wadt, W. R., J. Chem. Phys. 1985, 82, 270.; Wadt, W. R.; Hay, P. J. J. Chem Phys. 1985, 82, 284.; Hay, P. J.; Wadt, W. R. J. Chem. Phys. 1985, 82, 299. 
v. Höllwarth, A.; Böhme, M.; Dapprich, S.; Ehlers, A. W.; Gobbi, A.; Jonas, V.; Köhler, K. F. Stegmann, R.; Veldkamp, A.; Frenking, G. Chem. Phys. Lett. 1993, 208, 237. 